\title{
Numerical Investigation of Marine Propeller Underwater Radiated Noise Using Acoustic Analogy \\ Part 1: The influence of grid resolution \\ Savas Sezen ${ }^{1 *}$, Taner Cosgun ${ }^{2}$, Ahmet Yurtseven ${ }^{2}$, Mehmet Atlar ${ }^{1}$ \\ ${ }^{1}$ University of Strathclyde, Glasgow, UK \\ ${ }^{2}$ Yildiz Technical University, Istanbul, Turkey
}

\section{Abstract}

This study investigates the effects of grid resolution on hydroacoustic performance of the benchmark INSEAN E779A propeller operated in uniform flow, open water and non-cavitating conditions. In the numerical calculations, an incompressible hydrodynamic solver together with the porous FW-H (Ffowcs-Williams Hawkings) equation is used to predict the propeller URN (Underwater Radiated Noise). The first aim within this study is to explore the sensitivity of the grid resolution on the prediction of propeller hydroacoustic performance. Furthermore, amongst the contribution of nonlinear noise sources on overall acoustic pressure, the role of the tip vortex is believed to be dominant under non-cavitating conditions. The inadequate extension of the tip vortex is one of the drawbacks in the RANS (Reynolds-averaged Navier-Stokes) solver for accurate prediction of propeller URN, especially at the receivers located in the propeller's slipstream. Thus, the second aim within this study is to examine the contribution of tip vortex on overall acoustic pressure. In order to visualise the numerical noise and determine the realistic extension of the vortex distributions in the propeller's slipstream, the time derivative of the pressure technique is proposed in this study. The results indicate that insufficient grid resolution in the numerical simulations causes unphysical numerical noise which is attributed to the sliding interfaces, and it leads to contamination of the overall acoustic pressures. Moreover, an increase in grid resolution reduces numerical diffusion in the RANS solver, allowing for an extended tip vortex distribution. However, an increase in tip vortex extension and intensity alongside the downstream of the propeller is not adequate itself to make a reliable prediction of propeller URN by using RANS. Consequently, a more realistic prediction of propeller URN requires the use of advanced models (i.e. LES (Large Eddy Simulation) and DES (Detached Eddy Simulation)) together with the porous $\mathrm{FW}-\mathrm{H}$ equation, particularly if the receivers located in the downstream are of great interest.

Keywords: Hydroacoustic, FW-H, RANS, Grid Resolution, URN, Numerical Noise, E779A. 


\section{Introduction}

URN has become a significant issue in the last few decades because of the substantial increase in shipping noise in the world oceans. The studies revealed that an increase in URN induced by the shipping activities in the frequency range from $10 \mathrm{~Hz}$ to $1 \mathrm{kHz}$ is considered to be a potential threat to the marine ecosystem (Hildebrand, 2009; Urick, 1983). Due to this fact, great attention has moved to investigate the environmental effects of the noise induced by commercial vessels (Merchant et al., 2012). In this regard, international associations, classification societies, and conversation groups have urged the industry to address the harmful effects of noise pollution on marine fauna and take precautionary measures on this issue (Kellett et al., 2013).

In recent years, noise mitigation techniques have gained considerable importance with the aims to reduce levels by $3 \mathrm{~dB}$ in 10 years and $10 \mathrm{~dB}$ in 30 years (IWC, 2009). Furthermore, the International Maritime Organization (IMO) has pointed out this issue as a high priority and published non-mandatory guidelines to reduce URN induced by commercial ships (IMO, 2014). Despite the fact that several methods have become widespread in order to tackle this issue in the design stage of ships and mitigate the noise levels as much as possible, the hydrodynamic efficiency of a ship is usually considered a priority in comparison to the hydroacoustic performance. Therefore, this subject remains a primary deficiency of the shipbuilding industry, which might cause critical problems in the near future (Ianniello and De Bernardis, 2015).

A ship represents a very complex noise source, which constitutes of machinery noise, hydrodynamic flow noise and propeller radiated noise (ITTC, 2014a). Amongst the different noise sources, the propeller is an important one both under non-cavitating and cavitating conditions. In the presence of cavitation, pressure amplitudes increase rapidly, which results in higher noise levels at low and high-frequency parts of the noise spectrum. Besides, the propulsion system acts as both, a direct and indirect noise sources, triggering the stern of the hull. Thus, the stern part of the hull scatters the pressure itself and radiates noise under noncavitating conditions (lanniello et al., 2012).

The propeller URN can be predicted by using numerical methods, empirical formulations, and experiments under non-cavitating and cavitating conditions. Amongst the different methods, numerical prediction of the propeller URN by using viscous or potential based solvers together 
with the acoustic analogy is a relatively new research field for hydroacoustic studies. In fact, two different techniques can be employed, namely direct approach (i.e. Direct Numerical Solution (DNS)) and a hybrid method (i.e. hydrodynamic solver with the acoustic analogy) for the prediction of the URN. In the DNS method, the governing equations of the flow field and all turbulent scales are directly solved to compute the sound; thus, it makes the solutions more computationally expensive. Moreover, any direct method needs a compressible flow solution to compute the sound field. The sound propagates in a medium with a finite speed under the isentropic flow hypothesis (i.e. $c_{0}{ }^{2}=d p / d \rho$ ); hence, incompressibility assumption (i.e. $d \rho=0$ ) removes the propagation phenomena due to the infinite sound speed (i.e. $c_{0}=\infty$ ). Therefore, the DNS method is not appropriate for hydroacoustic calculations (lanniello et al., 2013a).

On the other hand, an alternative and more commonly used approach in hydroacoustic field is a hybrid method, which consists of an incompressible hydrodynamic solver together with the acoustic analogy. In this way, source and propagation fields can be decoupled. The source field is first determined by using an incompressible flow solver, whereas the propagation of the sound is provided by the aid of acoustic analogies from near field to far-field as a transfer function. The widely used acoustic analogy is Ffowcs-Williams Hawkings (FW-H) equation both in the aeroacoustics and hydroacoustic fields (Ffowcs Williams and Hawkings, 1969). The FW-H acoustic analogy, which is coupled with different hydrodynamic solvers (e.g. potential or viscous based solvers), have been commonly used for the prediction of propeller URN under non-cavitating and cavitating conditions in the literature (e.g. Bensow and Liefvendahl, 2016; lanniello et al., 2013a; lanniello et al., 2013b; lanniello et al., 2013c; Kellett et al., 2013; Lidtke et al., 2015; Lloyd et al., 2014; Lloyd et al., 2015a; Lloyd et al., 2015b; Seol et al., 2002; Seol et al., 2005; Sezen et al., 2020; Testa et al., 2008).

The propeller URN studies were firstly conducted using potential based solvers together with the acoustic analogy under non-cavitating and cavitating conditions. In this way, the feasibility of the acoustic analogy, main numerical issues and pros \& cons of the acoustic analogy have been examined via the potential based solvers in the literature. Seol et al. (2002) conducted a numerical study for the prediction of non-cavitating propeller URN by utilising the $\mathrm{FW}-\mathrm{H}$ equation, which is coupled with BEM (Boundary Element Method) solver, for a wide range of operating conditions. One of the aims of the study was to investigate the effects of duct 
geometry on overall URN by taking sound reflection and scattering effects into account. The results showed that the influence of duct geometry on overall URN is small in the far-field since the same directivity pattern was observed w/o ducted propellers. Seol et al. (2005) extended their previous study and investigated the propeller URN in the presence of sheet cavitation. In their study, the high-frequency part of the noise was left out. It was considered that the modelling of real cavity collapses and shock is too complex and dominated by nonlinearities. As a result of the study, the noise characteristics of the propeller were presented for different conditions and the dominant noise source was determined. Testa et al., (2008) investigated the hydroacoustic performance of a marine propeller by using a different numerical approach. The applications of FW-H acoustic analogy and Bernoulli-based methodology were discussed for naval applications. The effects of wake modelling and incompressibility assumption on propeller hydroacoustic performance were also examined. It was shown that $\mathrm{FW}-\mathrm{H}$ acoustic analogy is more robust and attaining many advantages in comparison to Bernoulli-based method. Seol (2013) proposed a new time-domain prediction method together with the vortex lattice method for the prediction of propeller URN in the presence of sheet cavitation. The numerical results were compared with the experimental data and those of potential-based numerical prediction method. The results revealed that pressure fluctuation, which occurs due to the sheet cavitation, is not simply proportional to the second derivative of the cavitation volume variation and inversely proportional to receiver distance. Testa et al. (2018) presented a novel hydroacoustic formulation for the prediction of propeller tonal noise in the presence of unsteady sheet cavitation and nonhomogeneous onset flow. The results of the novel formulation were compared with those of alternative numerical approach in the literature. It was found that new formulation gave more accurate results than the alternative approach in terms of noise magnitude and directivity.

In addition to the studies, which have been conducted using potential based solvers together with the acoustic analogy, the application of viscous based solvers became popular and widely used for the hydroacoustic problems in the maritime field utilising the development of computer technology. As a consequence, the hydrodynamic flow field is analysed through the RANS, DES or LES methods and then time-dependent variables are provided as an input to the acoustic analogy for the prediction of URN both in near and far-fields. Ianniello et al. (2012) explored the non-cavitating propeller hydroacoustic performance by using RANS with 
different resolution forms of the $\mathrm{FW}-\mathrm{H}$ equation (i.e. impermeable and permeable), both in the isolated case and a complete ship model. The contribution of linear and nonlinear noise terms on overall acoustic pressure was explained in detail. The numerical results showed that URN is considerably affected by the contribution of nonlinear noise sources and they must be included in the calculations for the reliable prediction of propeller URN. Later on, the authors conducted a comprehensive study for the prediction of INSEEAN E779A propeller hydroacoustic performance under non-cavitating conditions (lanniello et al., 2013a). Timebased acoustic pressure signals were derived at different loading conditions and compared with the hydrodynamic pressures. The results showed that quadrupole noise terms could not be neglected regardless of the propeller rotational speed for the hydroacoustic calculations, unlike the aeroacoustics field. Ianniello and De Bernardis (2015) investigated the hydroacoustic performance of INSEAN E779A model propeller in uniform flow by using RANS and DES solvers together with FW-H equation. In the numerical calculations, the RANS solver exhibited three subsequent spirals of tip vortex in the propeller's wake before fading, whereas DES showed persistence tip vortex distribution in the propeller's slipstream. Thus, it was considered that RANS solvers became inadequate for hydroacoustic purposes, particularly for the receivers where the turbulent fluctuating component of the velocity field is relevant. Lloyd et al. (2014) compared two different numerical solvers (i.e. ReFRESCO with porous FW$\mathrm{H}$ and EXCALIBUR with Kirchhoff formulation) for the two-bladed model propeller (i.e. S6666) in open water condition. The main aim of the study was to verify the FW-H analogy and investigate the behaviour of the porous surface at different receiver locations. The results showed that $\mathrm{FW}-\mathrm{H}$ results are in good agreement with the measurement data, but $1^{\text {st }} \mathrm{BPF}$ value was underpredicted. In contrast, Kirchhoff formulation showed slightly better agreement with the measurements than the $\mathrm{FW}-\mathrm{H}$ solution for a specific porous data surface. Lloyd et al. (2015a) investigated capabilities of the acoustic analogy together with the RANS solver in the near field for different grid resolutions. In the numerical calculations, both steady and unsteady approaches were used to compute the acoustic pressures for the receivers located at the propeller plane. The numerical results showed that both unsteady RANS and $\mathrm{FW}-\mathrm{H}$ pressures deteriorate due to some numerical disturbances, which might be associated with the sliding interface or pressure correction methods. Lloyd et al. (2015b) also examined the propeller hydroacoustic performance using RANS together with porous $\mathrm{FW}-\mathrm{H}$ formulation with two different CFD codes (i.e. ReFRESCO and OpenFOAM). In the author's study, the 
effects of permeable surface closure on the propeller hydroacoustic performance were evaluated at two receivers, which were located in the propeller's slipstream. The results revealed that the acoustic pressures at the receivers, which are close to the end-cap of the porous surface, were more sensitive to the inclusion of downstream side end-cap. Additionally, it remained unclear whether considerable contribution for the receivers in the propeller's slipstream is due to the nonlinear sources or not. Testa et al. (2018) compared DES and BEM solvers, which are coupled with a porous FW-H equation, for the INSEAN E779A propeller under uniform and non-cavitating conditions. The main purpose of the study was to present the capabilities of the BEM solver for propeller URN in open water conditions. The results showed that important vorticity field in the propeller's slipstream characterises the overall sound pressure level in DES solver. Yet, BEM solver was found to be inadequate to reflect the effects of nonlinear noise sources as moving downstream of the propeller. Hence, BEM solver was found to be inappropriate for more in-depth hydroacoustic investigations. Cianferra et al. (2019) focused on the hydrodynamic noise radiated by a ship propeller in open water and non-cavitating conditions. In the numerical calculations, LES solver together with FW-H equation was utilised. In the authors' study, different noise generation mechanisms were examined separately. As a result of the study, it was found that the nonlinear quadrupole term dominates broadband noise spectrum and the contribution of shaft vortex on overall URN was found to be higher for far-field noise predictions. Lidtke et al. (2019) focused on hydroacoustic performance prediction of INSEAN E779A model propeller under non-cavitating and cavitating conditions in the presence of wake field by using RANS together with the FW-H acoustic analogy. The authors' study might be the first study to test the capabilities of the acoustic analogy in a realistic test configuration in the hydrodynamic field. The main purpose of the study was to explore the crucial solution parameters (e.g. time step, grid resolution and sensitivity of porous data surface) on the prediction of propeller URN. The result revealed that larger porous data surface in the coarse grid region might risk information loss due to the discretisation errors and dissipation.

In addition to the studies, which were conducted to predict propeller URN, the scattering problem, which occurs due to the periodic load induced on the hull and its appendages by the propeller, has been investigated in the literature. The acoustic waves directly generated by the marine propeller interferes with the scattered pressure from the hull and cause change 
in near and far-field noise predictions. Since the 1980s, different numerical methods and solution methodologies have been developed to compute the scattering of acoustic waves from different objects (Kehr and Kao, 2011). For this reason, Kehr and Kao (2011) developed a numerical method to predict the underwater acoustic field and pressure fluctuation on a ship hull in the presence of unsteady propeller sheet cavitation by using linear wave equation. The pressure field, which is radiated directly from the propeller and scattered from the ship hull with the free surface effects, were included to the calculations. The results were compared with those of Laplace equation and experimental data. The difference between the authors 'method and experiment was stipulated to be because of the contribution of tip vortex cavitation, which was neglected in the authors' study. lanniello et al. (2013b) implemented acoustic analogy to predict the URN for a complete scaled ship model. In the numerical calculations, the RANS method was used together with $\mathrm{FW}-\mathrm{H}$ equation. The hydrodynamic and hydroacoustic solutions indicated that there is a discernible scattering effect due to the hull surface. The results showed that the large contribution of loading noise term from the hull scattered pressure on the overall acoustic pressure was observed due to the unsteady load on the hull induced by propellers. Wei et al. (2016) predicted the noncavitating propeller noise in the wake of a submarine by taking the scattering effects caused by submarine's hull into account using an effective iteration method based on the Helmholtz integral equation. The results showed that propeller noise levels increase due to the scattering effect from approximately $3 \mathrm{~dB}$ to $20.2 \mathrm{~dB}$ for $1^{\text {st }}, 2^{\text {nd }}$, and $3^{\text {rd }}$ BPF values. Testa and Greco (2018) examined the non-cavitating propeller tonal noise scattered by the submarine hull. The incident pressure field induced by a propeller on the scatterer hull surface was detected by the unsteady three-dimensional panel method together with the Bernoulli equation. It was stated that the proposed scattering formulation is suitable to compute the noise magnitude and directivity both on the hull and in the flow field.

All studies given above investigated the propeller URN by using $\mathrm{FW}-\mathrm{H}$ acoustic analogy together with both potential and viscous based solvers under non-cavitating and cavitating conditions. The contribution of non-linear noise sources (i.e. quadrupole noise term), key simulation parameters, noise directivities as well as scattering effects were investigated at different operating conditions. Unlike the aeroacoustics field, it has been shown that the contribution of nonlinear noise terms, which are mainly represented by vorticity and 
turbulence, cannot be neglected for reliable hydroacoustic predictions, particularly under non-cavitating conditions (Ianniello et al., 2013a). For this reason, a numerical solution of the flow field around the marine propeller is vital to provide the required data to the acoustic analogy. The flow field around the propeller can be solved either by using RANS solver or scale resolving simulations (i.e. DES or LES). The capabilities and sensitivities of the acoustic analogy have been commonly investigated with the RANS solver, particularly in the preliminary design stage. Inevitably, DES and LES improve the reliability of the solution and take more details of the unsteady flow field into account. However, the application of the advanced models is more computationally expensive than the RANS solver. Hence, RANS solver becomes appealing and it is still being used in the literature for the investigation of crucial simulation parameters, which are mainly associated with timestep and grid resolution, and sensitivity of the acoustic analogy (e.g. Lidtke et al., 2019). Amongst the important solution parameters, the grid should be designed to improve the accuracy of the hydrodynamics inputs and mitigate the non-physical numerical noise, which might deteriorate the overall acoustic pressure. As stated in the study of Lloyd et al. (2015a), the $\mathrm{FW}-\mathrm{H}$ equation is more sensitive to hydrodynamic inputs; hence, grid resolution. Besides, the required grid resolution for the hydroacoustic simulations was also discussed in the $27^{\text {th }}$ ITTC Noise discussion Forum (ITTC, 2014b) and it was suggested to use high-quality grid structure for the reliable prediction of propeller URN. The necessity of more validation and verification studies was also emphasised for the application of acoustic analogy.

In this framework, the need to gain a deeper knowledge for the numerical noise issue in the numerical solvers, which can be mainly associated with the grid resolution, and understand the sensitivity of the acoustic analogy is the initial motivation of this study. In this regard, different grid resolutions were adopted to RANS solver; thus, change in the hydro dynamic and hydroacoustic pressures with different grid resolutions was examined at several receiver locations. Although there are different ways available in Star+ CCM (2019) to model the propeller motion (i.e. MRF, RBM and Chimera technique), only RBM (sliding interface) approach was utilised in the scope of this study. In order to observe the numerical noise, which is usually attributed to the sliding interfaces, the time derivative of the acoustic pressure technique was prosed in this study. Additionally, the same approach was also suggested for the accurate visualisation of vortex structures in the propeller's slipstream for hydrodynamic and hydroacoustic studies. Furthermore, the application of inadequate grid 
resolution in the numerical solver increases the numerical diffusion, particularly for RANS solver. As stated in several papers in the literature (e.g. Ianniello and De Bernardis, 2015; Muscari et al., 2013), the vortex structures released at the propeller blade's tip and hub disappear rapidly in the propeller's slipstream in the RANS solver. For this reason, an inadequate extension of the tip vortex downstream of the propeller is one of the drawbacks of the RANS solver aside from the poor modelling of turbulence for the hydroacoustic calculations, especially for the receivers located in the propeller's slipstream. Therefore, the contribution of tip vortex on overall acoustic pressure was investigated with an increase in grid resolution at the receivers located in the downstream.

The structure of this paper is organised as follows. Section 2 presents a summary of the hydroacoustic model. Description of the test case, computational domain \& boundary conditions and grid structure is explained in Section 3. Section 4 and 5 present the hydrodynamic \& hydroacoustic results and conclusion part, respectively.

\section{Theoretical Background}

The numerical background of this study is based on the Navier-Stokes equations. Here, only FW-H equation is introduced. The detailed information about the hydrodynamic model (i.e. RANS) can be found in the commercial solver guideline (Star CCM+, 2019).

\subsection{Hydroacoustic Model (FW-H Equation)}

Numerical CFD methods (i.e. potential or viscous based solvers) are used for the assessment of the flow field. Once the sound source is identified in the fluid domain, several acoustic analogies can be used for sound propagation from the near to far-field. Lighthill et al. (1952) pioneered the development of acoustic analogy by ignoring the presence of a solid surface in the flow field. Following this, a more general formulation was developed by Curle (1955) for the cases where a stationary solid surface is available in the flow field. Later on, the formulation was generalised by Ffowcs Williams Hawkings (FW-H) (Ffowcs Williams and Hawkings, 1969) for moving and permeable surfaces. In the numerical calculations, FW-H formulation has been generally applied as an integral method for the prediction of the noise in the far-field both in aeroacoustics and hydroacoustic fields (Lyrintzis, 2003; Najafi-Yazdi et al., 2010; Nitzkorski, 2015 )

The $\mathrm{FW}-\mathrm{H}$ equation is the rearrangement form of the continuity and momentum equations into wave equations (Ffowcs Williams and Hawkings, 1969). The integral solution of the FW- 
$\mathrm{H}$ equation is based on the use of Green`s functions. In this equation, three different noise generation mechanisms are present. The generalised formulation of the $\mathrm{FW}-\mathrm{H}$ equation is given as follows.

$$
\begin{aligned}
& \left(\frac{1}{c_{0}^{2}} \frac{\partial^{2}}{\partial t^{2}}-\nabla^{2}\right) p(x, t)=\frac{\partial}{\partial t}\left\{\left[\rho_{0} v_{n}+\rho\left(u_{n}-v_{n}\right)\right] \delta(f)\right\}- \\
& \frac{\partial}{\partial x_{i}}\left\{\left[\Delta P_{i j} n_{j}+\rho u_{i}\left(u_{n}-v_{n}\right)\right] \delta(f)\right\}+\frac{\partial}{\partial x_{i} \partial x_{j}}\left\{T_{i j} H(f)\right\}
\end{aligned}
$$

Here, first-term indicates thickness term (i.e. monopole); the second one is loading (i.e. dipole) term, and the latter one is the nonlinear (i.e. quadrupole) term at the right-hand side of Equation 1. Additionally, in Equation 1, $p$ identifies the acoustic pressure disturbance, $\nabla^{2}$ is Laplace operator, $\rho$ is density, $t$ is observer time, $x$ is location, $T_{i j}\left(T_{i j}=\rho u_{i} u_{j}+P_{i j}-\right.$ $\left.c_{0}^{2} \tilde{\rho} \delta_{i j}\right)$ is Lighthill stress tensor, $u_{i}$ and $v_{i}$ are the fluid and surface velocity components, respectively. $\tilde{\rho}=\rho-\rho_{0}$ is the density perturbation for the undisturbed medium, $n$ indicates the projection along the outward normal to the surface, $c_{0}$ is the sound speed, $P_{i j}$ represents the compressive stress tensor $\left(\Delta P_{i j}=P_{i j}-p_{0} \delta_{i j}\right) . \delta$ is the Kronecker operator, $H$ is a Heaviside function.

If the impermeable condition is imposed on the calculations (i.e. $u_{n}=v_{n}$ ), a generalised formulation of $\mathrm{FW}-\mathrm{H}$ equation (i.e. Equation 1 ) simplifies and two linear terms can be written in the integral form. This form of the FW-H equation is known as Farassat $1 \mathrm{~A}$ (Farassat, 2007) solution. The impermeable form of the FW-H equation is commonly used in the aeroacoustics field. In contrast, as stated in the study of Ianniello et al. (2013a), the underwater field is considerably affected by the contribution of quadrupole noise sources in hydroacoustic applications; thus the porous or permeable type of the FW-H equation can be used effectively to include the nonlinear contributions. The porous formulation, which was applied by Ffowcs Williams and Hawkings (1969) in their original study and also proposed as a possible numerical solution of the FW-H equation by Di Francescantonio (1997), was used in this study. The main reasoning of this originates from the fact that directly computing the quadrupole noise terms are more computationally expensive and more sensitive to numerical errors.

In the porous formulation of $\mathrm{FW}-\mathrm{H}$ equation, an integral domain (i.e. porous or permeable surface) is defined around the body (i.e. around the propeller blades) to include all relevant 
nonlinear contributions as much as possible for the calculations. When porous formulation of $\mathrm{FW}-\mathrm{H}$ is used, the nonlinear terms inside the integral domain are evaluated by solving the surface integrals instead of the volume integral. In this way, the thickness and loading terms lose their physical meanings in the impermeable approach and became pseudo thickness and pseudo loading noise terms. In this formulation, two new variables $\left(U_{i}\right.$ and $\left.L_{i}\right)$ as modified velocity and modified stress tensor are defined as follows.

$U_{i}=u_{i}+\left(\frac{\rho}{\rho_{0}}-1\right)\left(u_{i}-v_{i}\right)$

$L_{i}=P_{i j} \hat{n}_{j}+\rho u_{i}\left(u_{n}-v_{n}\right)$

The generalised FW-H equation can be rearranged by changing $u_{n}$ and $P_{i j}$ with $U_{i}$ and $L_{i}$, respectively. The porous formulation of $\mathrm{FW}-\mathrm{H}$ equation can be written in Equation 4 for stationary integral domain, incompressibility and $M=|v| /\left|c_{0}\right| \ll 1$ (i.e.Mach Number) assumptions (Di Francescantonio, 1997).

$4 \pi p(x, t)=\int_{S} \frac{\rho_{0} \dot{U}_{n}}{r} d S+\int_{S} \frac{\dot{L}_{r}}{c_{0} r} d S+\int_{S} \frac{L_{r}}{r^{2}} d S+p_{Q}(x, t)$

Here, $r(m)$ is the distance between the noise source and the receiver, subscripts $r$ and $n$ define the dot product of a quantity with a unit vector in either radiation or normal directions, respectively. The last term $\left(p_{Q}(x, t)\right)$ still represents the nonlinear contribution of the noise sources where outside of the integral surface in the fluid domain. Similar to other studies in the literature (e.g. Ianniello et al., 2013a; Lidtke, 2017; Lloyd et al., 2015a), the noise contribution from the outside of the integral domain was neglected in this study. The reason behind this, the extension of the integral surface was far enough and included nearly all relevant nonlinear noise sources (i.e. contains all meaningful non-linearities).

\section{Numerical Modelling and Test Case Description}

\subsection{Propeller Geometry}

The numerical calculations were conducted by using a well-known model scale INSEAN E779A propeller, which is widely used both in hydrodynamic and hydroacoustic fields. The experimental hydrodynamic data is also available for the selected propeller (Salvatore et al., 2006). 3D view of the propeller and its main parameters are indicated in Figure 1 and Table 1 , respectively. 


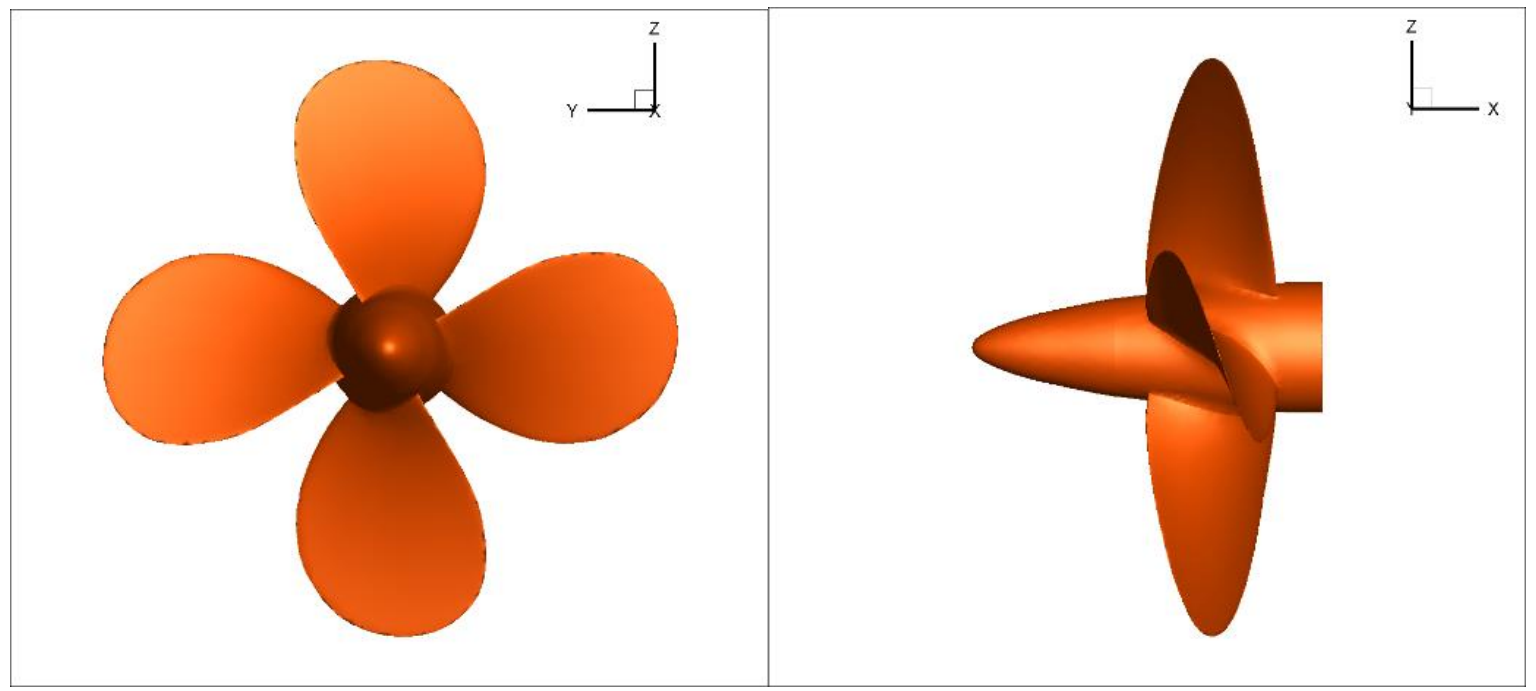

Figure 1. Back and side views of the INSEAN E779A model propeller.

Table 1. Main particulars of the propeller.

\begin{tabular}{|l|l|l|}
\hline Number of Blades & $Z$ & 4 \\
\hline Diameter $(\mathrm{m})$ & $\mathrm{D}$ & 0.227 \\
\hline Pitch Ratio & $\mathrm{P} / \mathrm{D}$ & 1.1 \\
\hline Expanded Area Ratio & $\mathrm{A}_{E} / \mathrm{A}_{0}$ & 0.69 \\
\hline
\end{tabular}

In the numerical calculations, the same operational condition (i.e. $J=0.88$ and $n=25 \mathrm{rps}$ ) was selected with those of other studies in the literature in order to compare the numerical results (e.g. Ianniello et al., 2013a; Lidtke, 2017; Testa et al., 2018). It should be pointed out that experimental URN data is not available for the selected propeller in the current literature.

\subsection{Computational Domain \& Boundary Conditions}

The computational domain and boundary conditions are given in Figure 2 . The computational domain was extended by 7D in the downstream of the propeller from the propeller blades centre, which enables wake evolution before reaching the outlet. Additionally, the computational domain was extended by $4 \mathrm{D}$ both in the upstream and radial directions from the propeller centre. The transition between the rotating and static regions was provided by a rotating interface (i.e. sliding interface). 

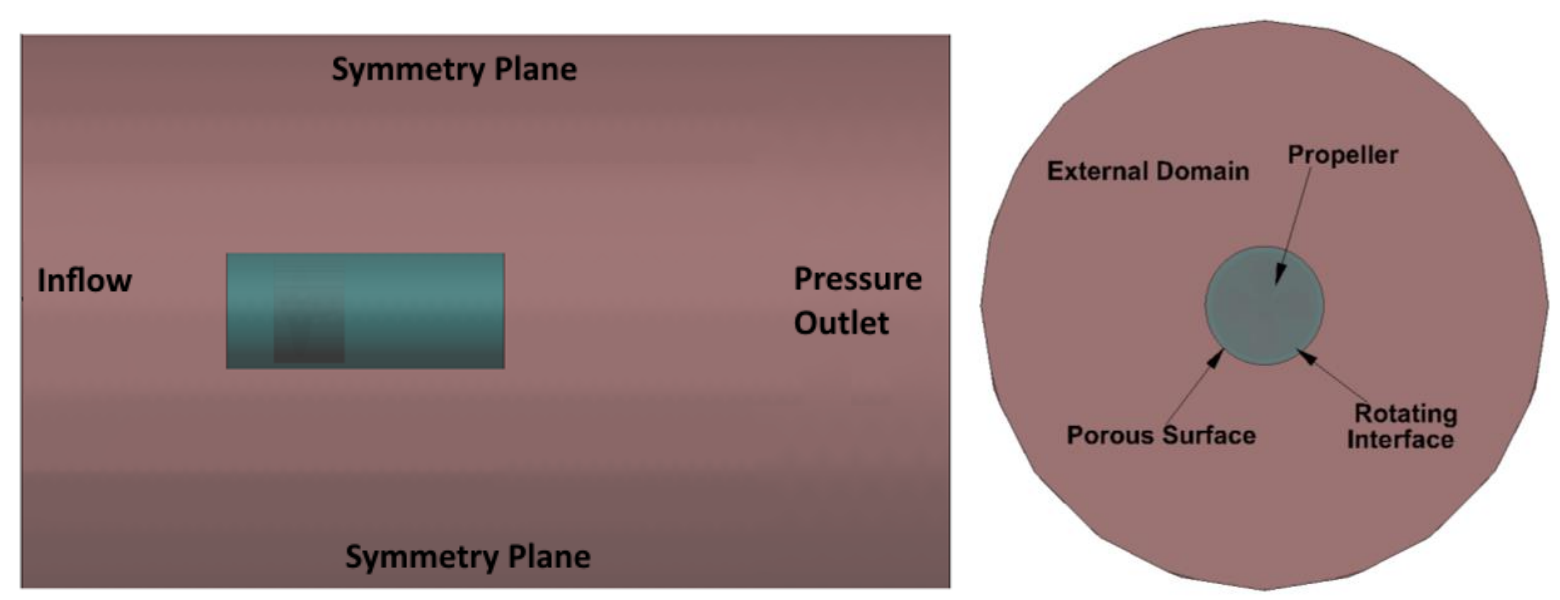

Figure 2. The computational domain used in the numerical calculations (Figure is not scaled).

In the numerical calculations, an integral surface (i.e. porous surface) was used to compute the hydroacoustic pressures at different receiver locations. As seen in Figure 3, the integral surface consists of the main cylinder and two end-caps (i.e. upstream and downstream endcaps). As expected, the downstream end-cap of the integral surface cuts the tip vortex and changes the overall acoustic pressure behaviour. Therefore, an open-ended integral surface, which was strongly proposed in the study of Ianniello and De Bernardis (2015), was used in the present study.

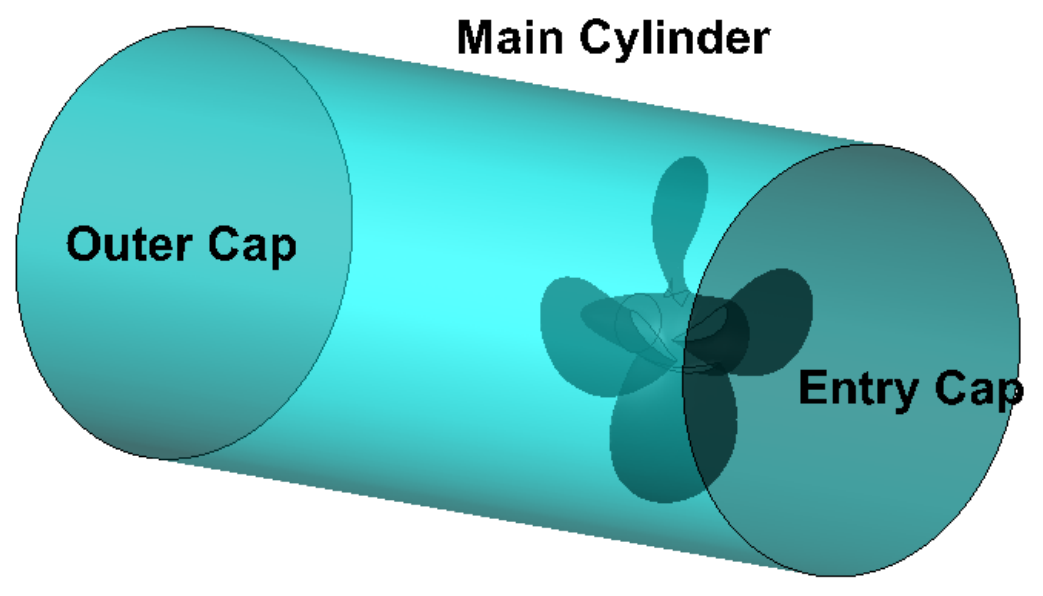

Figure 3. Representation of integral (i.e. porous) FW-H surface.

The exact dimension and location of the integral surface has not been defined clearly in the present literature and it is still being investigated (Bensow and Liefvendahl, 2016; Li et al., 2018; Lidtke et al., 2019). Nevertheless, the convergence study was conducted by varying the length and dimensions of the integral surfaces in this study. Table 2 summarises the geometrical properties of the integral surfaces. It should be noted that the dimensions of the 
base integral surface, which was used in this study, was set according to similar studies in the literature for the comparison of the numerical results (e.g. Ianniello et al., 2013a; Lidtke, 2017; Lloyd et al., 2015a).

Table 2. Geometrical features of the integral surfaces

\begin{tabular}{|c|c|c|}
\hline $\mathrm{ID}$ & $L / D$ & $D_{\text {integral }}$ \\
\hline 1 & 2.5 & $1.23 \mathrm{D}$ \\
\hline Base (2) & 3.0 & $1.25 \mathrm{D}$ \\
\hline 3 & 3.5 & $1.28 \mathrm{D}$ \\
\hline 4 & 4.0 & $1.32 \mathrm{D}$ \\
\hline
\end{tabular}

\subsection{Grid Structure}

Hydroacoustic simulations are deemed to be more sensitive to mesh quality and density than the traditional hydrodynamic simulations. Hence, a suitable grid structure should be adopted to increase the accuracy of the URN predictions. The applied grid structure should meet two main principles (Star CCM+, 2019);

- The grid should be adequate to resolve/model the sound generating turbulence scales, particularly in the propeller's slipstream where the contributions of nonlinear noise sources are dominant over blade dynamics.

- The grid should be able to resolve propagation of the acoustic waves towards the locations of the receivers.

In this study, the unstructured grid was used to discretise the computational domain within the facilities of Star CCM+ (2019). The region-based mesh technique was implemented with the cells, which have minimal skewness, by using the trimmer mesh algorithm. In this way, the higher numerical dissipation was prevented, and the accuracy of the solution was increased with a reasonable computational cost. The hexahedral elements were used for the discretisation of the computational domain as the application of the tetrahedral cells can deteriorate the accuracy of the solution (Jones and Clarke, 2010). Besides, during the mesh adaptation process, the cell sizes inside the integral surfaces were kept constant to prevent the additional non-physical noise, which can contaminate the overall acoustic pressures (Star $\mathrm{CCM}+, 2019)$. Cells inside the noise source region were aligned at the interfaces to facilitate the smooth transition between two regions. Besides, the grid quality at the interfaces was increased by identifying a layer of prismatic cells to reduce the numerical noise as much as possible. Moreover, almost uniform cell size, particularly in the $\mathrm{X}$ direction, was used to 
reduce the numerical diffusion. Different integral surfaces were placed inside the finer grid resolution to minimise the information loss.

Table 3 presents the different grid resolutions, which are generated to investigate both the numerical noise and contribution of tip vortex on overall acoustic pressure. The general view of the finest grid resolution is given in Figure 4.

Table 3. Total element counts of different grid resolutions ( $\mathrm{N}$; millions of cell)

\begin{tabular}{|c|c|}
\hline Grid & $\mathbf{N}$ \\
\hline Coarse & 2.8 \\
\hline Medium & 4.9 \\
\hline Fine & 10.3 \\
\hline Finer & 17 \\
\hline Finest & 31.6 \\
\hline
\end{tabular}
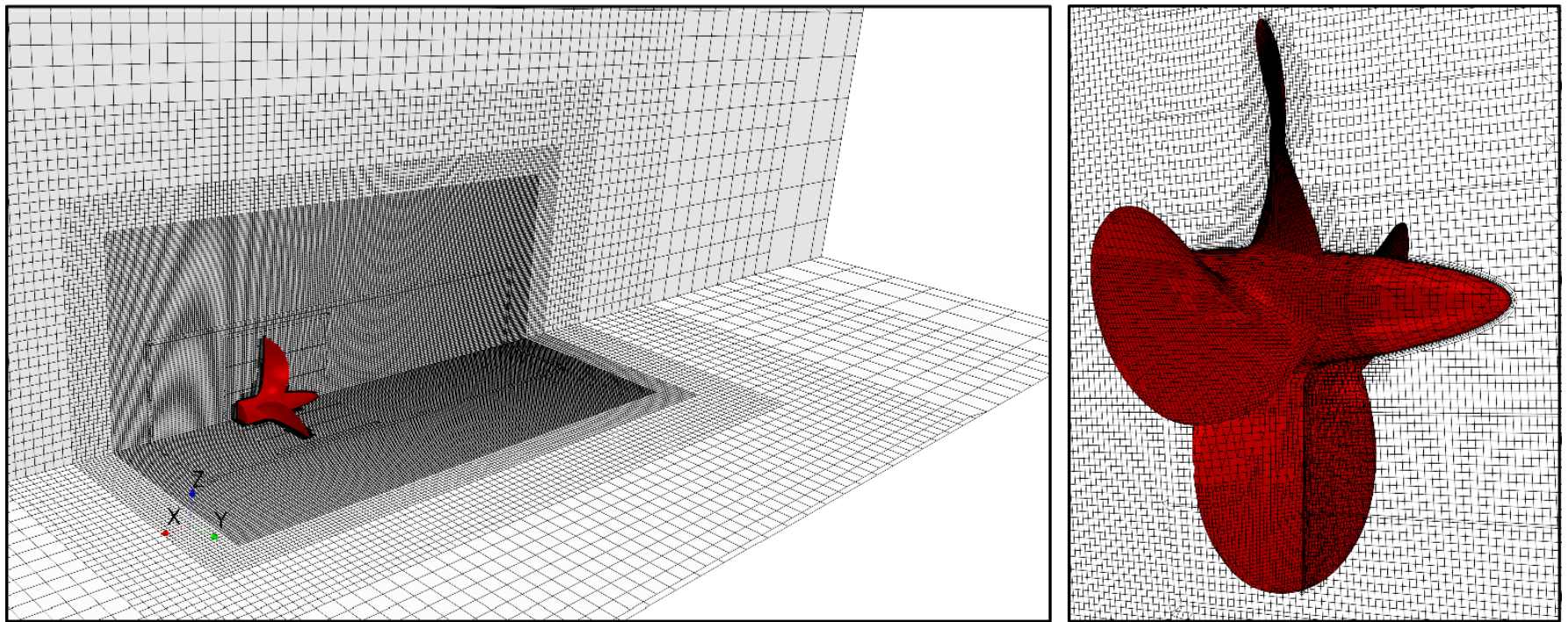

Figure 4. General view of the grid structure around the propeller blades (Finest Grid).

\subsection{Solution Strategy}

In the numerical calculations, RANS equations were discretised using the finite volume method. During the simulations, commercial CFD solver Star CCM+ was utilised (Star CCM+, 2019). The near wall-boundary layer was solved by using wall function and the average wall $y+$ value was approximately 35 in the calculations for different grid resolutions. The convective and temporal terms in the momentum equations were discretised with $2^{\text {nd }}$ order scheme. The equations were coupled by using a segregated SIMPLE type solution algorithm. The k- $\omega$ SST (Shear Stress Transport) turbulence model, which is commonly used for propeller hydrodynamic and hydroacoustic simulations, was used for turbulence modelling (e.g. Lloyd et al., 2015a; Zhu, 2014). During the simulations, the inner iteration was set to 8 . The simulations were first initiated in a steady manner to accelerate the convergence. After that, 
the unsteady simulations were conducted with $1^{\circ}$ of propeller rotational rate. After 20 propeller revolutions, it reduced gradually to $5^{\circ}$ of propeller rotational rate to eliminate the high-frequency noise. Consequently, the acoustic pressures were collected at 15 propeller revolutions.

In this study, steady initialisation was conducted by MRF (Moving Reference Frame), whereas RBM (Rigid Body Motion, also known as Sliding Mesh) was used in unsteady simulations. In the RBM technique, unsteady governing equations are solved at every timestep. In contrast, unsteady components of the flow field are not considered in the MRF technique. Therefore, the RBM technique produces more accurate results. In addition to this, it has been widely used for hydrodynamic simulations when any unsteady flow features in the flow region are of great interest (Moussa, 2014).

\section{Results and Discussion}

\subsection{Hydrodynamic Results}

As a first validation of the numerical results with different grid resolutions, open water characteristics (i.e. thrust, torque and efficiency values) of the propeller were compared with the experimental data at $\mathrm{J}=0.88$. The non-dimensional thrust $\left(K_{T}\right)$, torque $\left(K_{Q}\right)$ and efficiency $\left(\eta_{0}\right)$ values are calculated as follows.

$K_{T}=\frac{T}{\rho n^{2} D^{4}}$

$K_{Q}=\frac{Q}{\rho n^{2} D^{5}}$

Here; $\rho$ is the water density $\left(\mathrm{kg} / \mathrm{m}^{3}\right), n$ is the propeller rotational speed $(r p s)$, and $D$ is the diameter of the propeller $(m), \mathrm{T}$ is thrust $(N)$, and $\mathrm{Q}$ is the torque (N.m).

$\eta_{0}=\frac{J}{2 \pi} \frac{K_{T}}{K_{Q}}$

Advance ratio $(J)$ is defined by:

$J=\frac{V_{A}}{n D}$

where $V_{A}$ is the average flow velocity at the propeller plane $(\mathrm{m} / \mathrm{s})$. The hydrodynamic results are given together with the experimental data at $\mathrm{J}=0.88$ for different grid resolutions in Table 4. The uncertainty study was conducted via GCl (Grid Convergence Index) method based on the Richardson extrapolation technique (Richardson, 1911) for thrust and torque values in Table 5. GCI method, which is recommended for CFD verification studies in the ITTC procedure 
(ITTC, 1999, 2008), was first proposed by Roache (1998) and applied in several studies in the literature. Here, full details of the methodology were not included. The details of the procedure implemented in this study can be found in Celik et al. (2008). The main steps of the method can be classified as below;

- Refinement factor ( $r$ ) was selected for different solutions.

- Difference between results of solution scalars $(\varepsilon)$ was calculated for thrust and torque.

- Solution type was determined with the convergence condition $(R)$, which is calculated as the ratio of solution scalars, in the $\mathrm{GCl}$ method.

- Convergence condition $(\mathrm{R})$ indicates the solution criteria, which can be classified into oscillatory, monotonic convergence or oscillatory and monotonic divergence. In our case, $R$ was calculated in the range of monotonic convergence $(0<R<1)$.

- As the last step, the numerical uncertainty of the solution was calculated.

Table 4. Computed performance coefficients for different grid resolutions

\begin{tabular}{|c|c|c|c|c|c|}
\hline \multicolumn{5}{|c|}{ Numerical Results } & \multicolumn{2}{c|}{ Difference (\%, EFD-CFD) } \\
\hline Grid & $\boldsymbol{K}_{\boldsymbol{T}}$ & $\mathbf{1 0}_{\boldsymbol{Q}}$ & $\boldsymbol{\eta}_{\mathbf{0}}$ & $\boldsymbol{K}_{\boldsymbol{T}}$ & $\mathbf{1 0}_{\boldsymbol{Q}}$ \\
\hline Coarse & 0.1532 & 0.3103 & 0.6915 & 1.46 & 1.74 \\
\hline Medium & 0.1531 & 0.3105 & 0.6906 & 1.39 & 1.80 \\
\hline Fine & 0.1527 & 0.3108 & 0.6881 & 1.12 & 1.90 \\
\hline Finer & 0.1525 & 0.3112 & 0.6863 & 0.99 & 2.03 \\
\hline Finest & 0.1524 & 0.3115 & 0.6852 & 0.93 & 2.13 \\
\hline Experiment & 0.151 & 0.305 & 0.718 & \multicolumn{1}{c}{} \\
\cline { 1 - 4 }
\end{tabular}

Table 5. Uncertainty values of the numerical solution.

\begin{tabular}{|c|c|c|c|}
\hline Results & (Finest-Finer-Fine) & (Finer-Fine-Medium) & (Fine-Medium-Coarse) \\
\hline$\% \mathrm{GCl}\left(\mathrm{K}_{\mathrm{T}}\right)$ & 0.07 & 0.25 & 0.37 \\
\hline$\% \mathrm{GCl}\left(10 \mathrm{~K}_{\mathrm{Q}}\right)$ & 0.12 & 5.20 & 5.52 \\
\hline
\end{tabular}

It could be concluded that, from the point of the global quantities view (i.e. thrust and torque values), the difference between numerical and experiment results was found to be quite good even if the coarse grid resolution was adopted in the numerical solvers. However, prediction of vortex structures in the propeller's slipstream with different grid resolutions was entirely different.

Figure 5 shows the vorticity distributions (i.e. $\omega_{Y}$ ) in the propeller's slipstream, which were obtained by experiment and numerical solver with different grid resolutions. As seen in Figure 5 , the tip vortex can be extended to the downstream of the propeller with an increase in grid resolution. In contrast, the extension of tip vortex disappears rapidly by using relatively coarse 
grid resolutions due to the numerical diffusion. Nevertheless, the accuracy of the flow details inside the vortex core might be still questionable for RANS solvers.
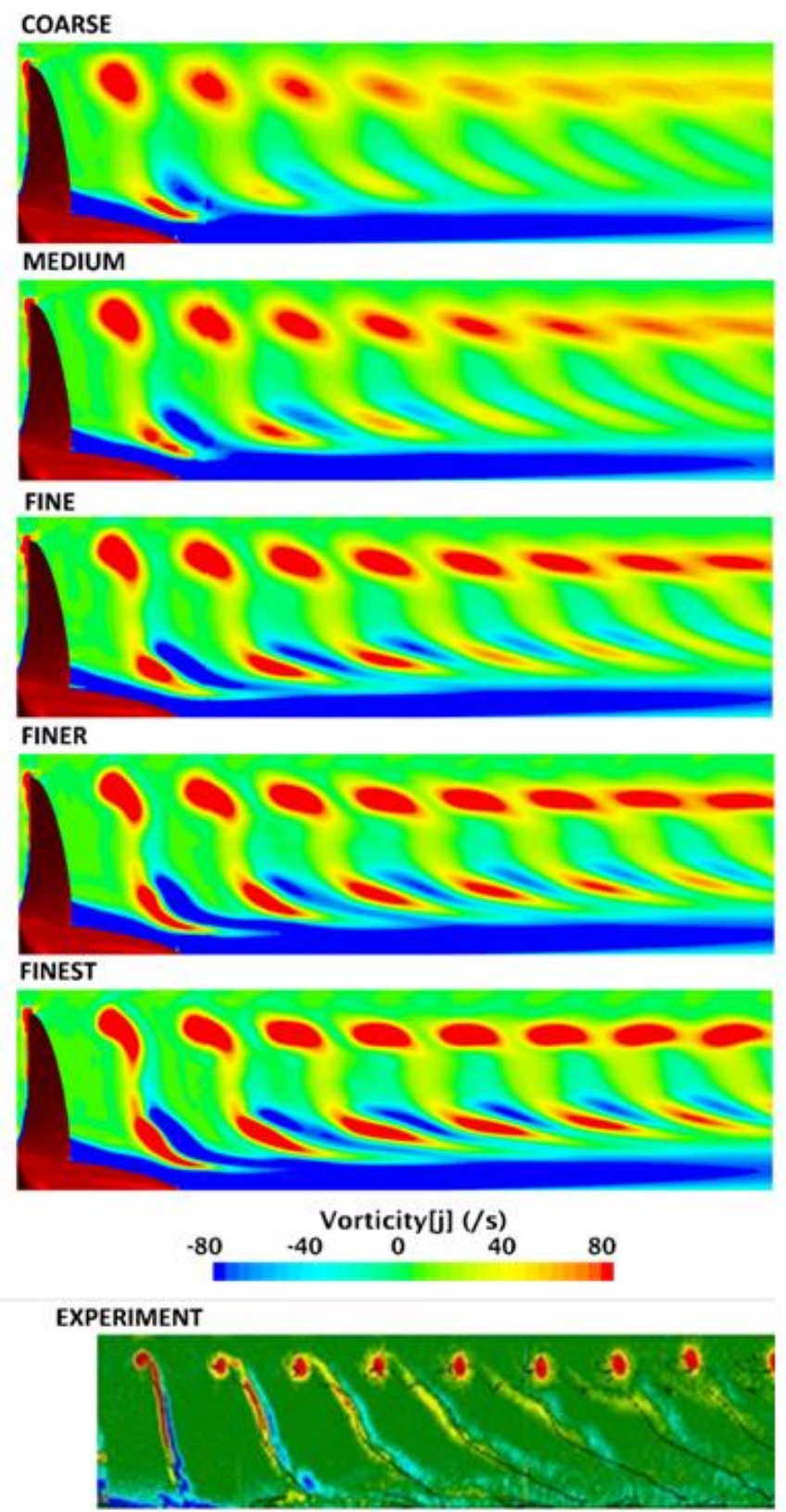

Figure 5. Comparison of the vorticity $Y$ component $\omega_{Y}$ with the experiment (Salvatore et al., 2006) on the plane $(y=0)$ for different grid resolutions 


\subsection{Hydroacoustic Results}

In this section, hydroacoustic results are presented to investigate the effects of grid resolution on propeller URN. The individual subsections are devoted to showing the numerical noise, which is associated with the sliding interfaces, and visualisation of the tip vortex in the propeller's slipstream. Furhermore, hydrodynamic and hydroacoustic pressures are compared at different receiver locations.

\subsubsection{Visualisation of numerical noise in hydroacoustic simulations}

The grid structure should be well designed in the numerical solver for the accurate calculation of hydrodynamic inputs; hence, propeller URN. Additionally, the numerical disturbances such as reflections from the domain boundaries, non-physical numerical noise generated by sliding (or rotating) interfaces, and inappropriate pressure correction methods can be considered key issues that require in-depth investigation. The artificial reflections created by the boundaries of the computational domain can be considerably reduced with the selection of suitable domain dimensions. It is to be noted that the present study only focuses on the investigation of the non-physical numerical noise induced by the sliding interface.

In the propeller hydrodynamic and hydroacoustic simulations, sliding interface mesh technique (i.e. RBM) is widely used to model the propeller's rotational motion. From the hydroacoustic point of view, one of the main disadvantages in this technique can be considered to cause additional numerical noise. The interface is mainly used to transfer the data and solution quantities such as pressure, velocity, temperature, etc. between the neighbouring regions. Thus, the suitable grid resolution is more important to increase the accuracy of the input data for hydroacoustic predictions. In particular, this issue might be more critical in the far-field where pressure fluctuations are smaller; hence, it may lead to pollution of the overall acoustic pressures. It should be pointed out that the numerical noise issue can also be faced at the interface of the integral (or porous) surface. However, as the static (i.e. non-rotating) porous surface was used in this study, any additional numerical noise was not observed from the interface of the integral surface (see Figure 8). In addition, as stated in Section 3, smooth mesh transitions were provided between inside and outside of the integral surface to minimise the numerical noise.

In this study, the numerical noise, which is associated with the sliding interfaces, was observed by using the time derivative of the pressure. For this purpose, a user-based field 
function and additional pressure monitor were introduced to the solver according to the following formulation.

$\frac{d P}{d t}=\frac{P^{n}-P^{n-1}}{\Delta t}$

Here, $P$ is acoustic or hydrodynamic pressures and $\Delta t$ is timestep. The pressure difference between consecutive time steps was computed by using the stored pressure value of every node point at the previous time step. Once the flow field converged, this function was activated to visualise the pressure variation with time in the flow field.

Coloured rectangles (i.e. blue and red regions) show the additional sources of sound-induced by the rotating interfaces for different grid resolutions in Figure 6 . Within the computational domain, there is no noise source that would cause rapid pressure variations on either side of the interface boundaries. Thus, acoustic pressure is expected to propagate towards the downstream of the propeller without any deterioration. However, the strong pressure fluctuations occurring at the interfaces can be proof of the non-physical numerical noise. As shown in Figure 6, even though an increase in grid resolution enables reduction in numerical noise at the sliding interfaces, it does not disappear entirely using the RBM method. Hence, it consequently might lead to a change in either amplitude or overall shape of the pressure signal. 


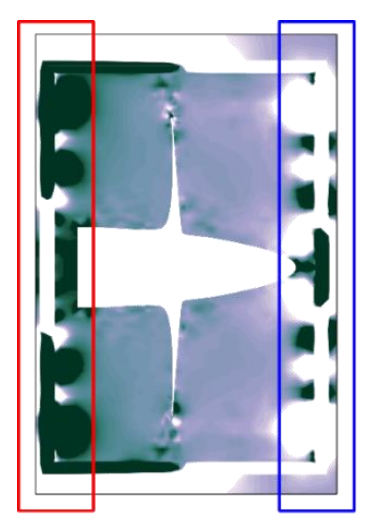

COARSE GRID

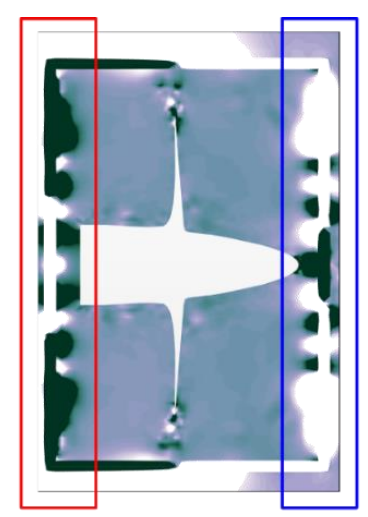

MEDIUM GRID

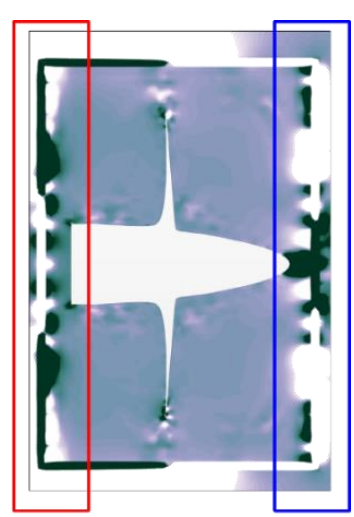

FINE GRID

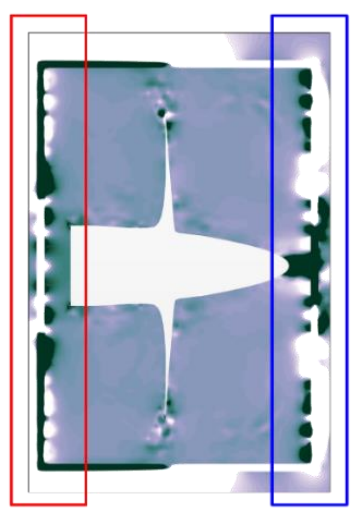

FINER GRID

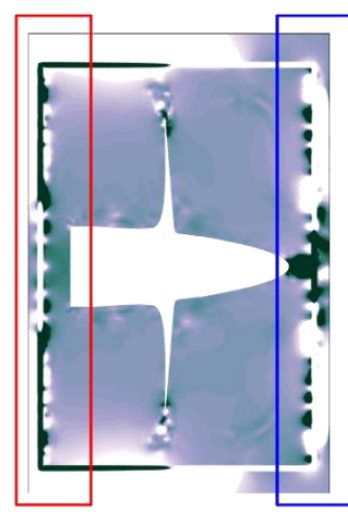

FINEST GRID

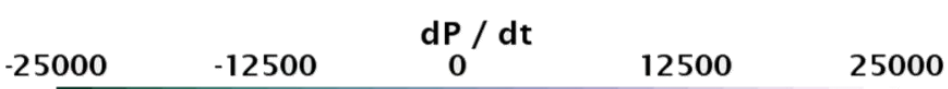

Figure 6. Numerical noise induced by rotating interfaces for different grid resolutions.

\subsubsection{Visualisation of tip vortex extension}

The coherent vortical structures in the propeller's slipstream are generally visualised in different numerical methods (e.g. RANS, DES and LES) by using either $Q$ or $\lambda_{2}$ criteria, which are based on the velocity gradient tensor. In this way, the comparison of the hub and tip vortices with the experiment can be performed to show the capabilities of the numerical solution and detailed investigation of the vortical structures in the wake of the propeller. In the numerical solver, $Q$ criterion is defined by the following formulation;

$$
Q=1 / 2\left[\left(|\Omega|^{2}-|S|^{2}\right)\right]
$$

Where $S$ donates the strain rate tensor and $\Omega$ is the angular rotation rate tensor (or vorticity tensor). According to $Q$ criterion magnitude, the dominant parameter in the flow field can be determined. When its value is positive, the flow field is dominated by the vorticity, whereas strain rate dominates the flow field when its value is negative (Star CCM+, 2019). Similar to $Q$-criterion, $\lambda_{2}$ criterion, which is based on the mathematical formulation proposed in Jeong 
and Hussain (1995), is another technique for detection of the vortex structures in the flow field. In this technique, the velocity gradient $(\mathrm{J})$ is first calculated and then it is assessed for each point of in the domain. It is decomposed as symmetric $(S)$ and anti-symmetric part (O) and defined as;

$$
\begin{aligned}
& S=\frac{J+J^{T}}{2} \\
& O=\frac{J-J^{T}}{2}
\end{aligned}
$$

Following the calculation of the symmetric and anti-symmetric part, a matrix is obtained with three eigenvalues. The value of the second eigenvalue shows the scalar quantity of $\lambda_{2}$. When the value of $\lambda_{2}$ is negative, the vortex structures can be detected in the flow region. Detailed information about the vortex identification methods can be found in the studies of Kolár (2007), Epps (2017) and Jeong and Hussain (1995).

In the numerical solvers, the visualisation of the vortex distribution in the propeller's slipstream strongly depends on the threshold value of the criteria (i.e.Q and $\lambda_{2}$ ), which results in a different prediction of tip and hub vortices extensions in the downstream of the propeller. However, an accurate prediction of the vortical structures extension in the wake of the propeller can be important for detailed investigation of the flow field. For this reason, the time derivative of the pressure technique, which was explained in Section 4.2.1, can also be used to determine the more realistic extension of the vortex distributions. As a spatial and temporal derivative of the pressure show the change in pressure with time and position in the flow field, different pressure fields are created around the vortex because of its physical dynamics (Hunt et al., 1988). Therefore, the tip and hub vortex regions can be first detected with the time derivative of the pressure technique on the longitudinal plane since the contribution of the tip vortex is dominant in the propeller's slipstream (Felli et al., 2006; Jang et al., 2001).

Figure 7 shows the change in extension of tip vortex distributions in the propeller's slipstream through the different threshold value of the $Q$ criteria and time derivative of the pressure technique. As can be seen in Figure 7, the location and extension of the pressure fluctuations do not change with the selection of different threshold values (e.g. 10000, 25000 and 50000) in the time derivative of pressure technique, while the pressures intensities in the vortex 
regions show a small variation. In contrast, the extension of the tip vortex changes significantly with the selection of various threshold values (e.g. 500, 1500, 3000) in $Q$ criterion. It consequently leads to misprediction of tip vortex extension in the numerical calculations. Therefore, time-derivative of the pressure technique can be reliably used to determine the exact location and extension of the tip vortex. This approach can also be used together with the $Q$ criterion for determination of the threshold value in the $Q$ criterion. In this way, the helical tip vortex can be observed.

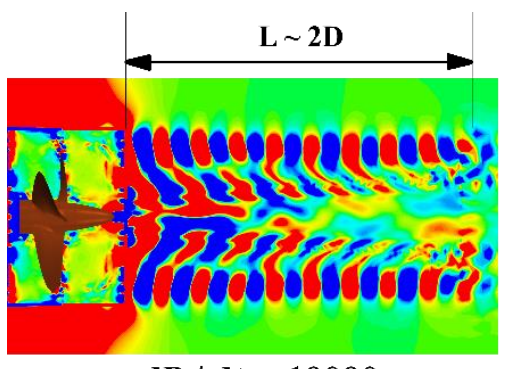

$\mathrm{dP} / \mathrm{dt}=\mathbf{1 0 0 0 0}$

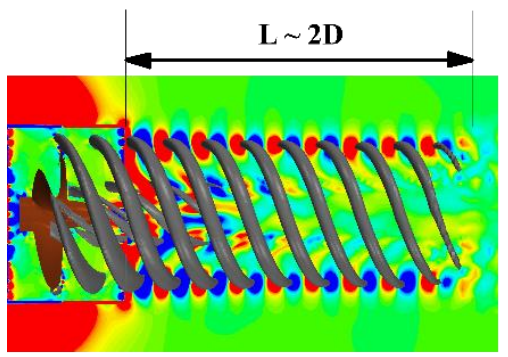

$\mathrm{dP} / \mathrm{dt}=\mathbf{2 5 0 0 0} \mid \mathrm{Q}, \mathrm{c}=\mathbf{5 0 0}$

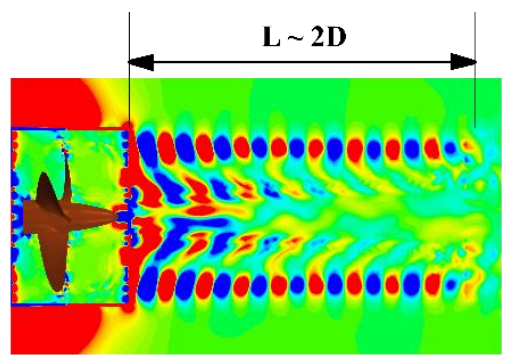

$\mathrm{dP} / \mathrm{dt}=\mathbf{2 5 0 0 0}$

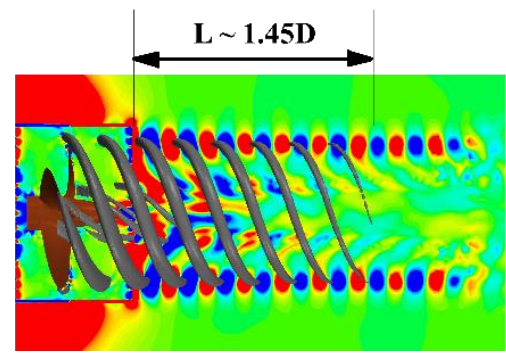

$\mathrm{dP} / \mathrm{dt}=\mathbf{2 5 0 0 0} \mid \mathrm{Q}, \mathrm{c}=\mathbf{1 5 0 0}$

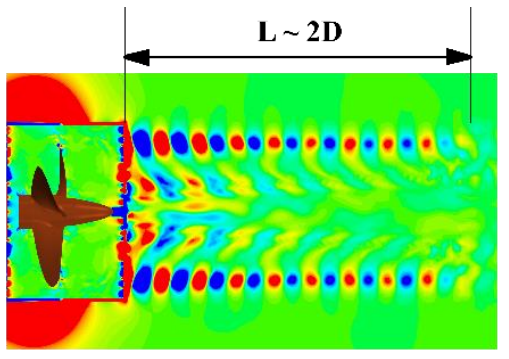

$\mathrm{dP} / \mathrm{dt}=\mathbf{5 0 0 0 0}$

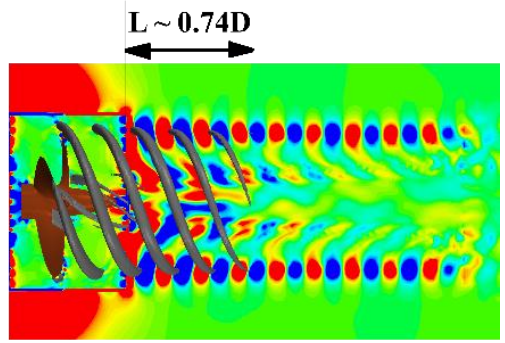

$\mathrm{dP} / \mathrm{dt}=\mathbf{2 5 0 0 0} \mid \mathrm{Q}, \mathrm{c}=\mathbf{3 0 0 0}$

Figure 7. Change in tip vortex extension with the time derivative of the pressure technique and $Q$ criterion (finer grid resolution).

In this study, the proposed technique was used to visualise the tip vortex in the propeller's slipstream for several grid resolutions in Figure 8. As known, the RANS method suffers from both turbulence modelling and discretisation errors; thus, it produces a higher amount of numerical diffusion. Due to this fact, the vortical structures in the propeller's slipstream disappear rapidly (Feder and Abdel-Maksoud, 2016; Duraisamy and Baeder, 2006). The numerical dissipation of the vortices can be deemed to be directly related to grid resolution and mainly occurs due to the insufficient discretisation of the nonlinear convective term (Ramponi and Blocken, 2012). However, this can be reduced with an increase in grid resolution in the numerical solvers and tip vortex can be further extended downstream of the propeller (Duraisamy and Baeder, 2006). 
Figure 8 indicates the tip vortex extension with different grid resolutions. As shown in Figure 8, a finer grid resolution results in a greater tip vortex extension in the propeller's slipstream. Furthermore, an increase in grid resolution changes the pressure field inside the tip vortex. The magnitude of the pressure is high in the vicinity of the propeller blade, whereas it reduces gradually as far away from the downstream, especially for coarse, medium, and fine grid resolutions. In contrast, the pressure inside the tip vortex reduces considerably for the finest grid resolution. Hence, an increase in grid resolution would also facilitate the observation of tip vortex cavitation in the propeller's slipstream since the main aim is to decrease the pressure below the saturation pressure inside vortex cores. 

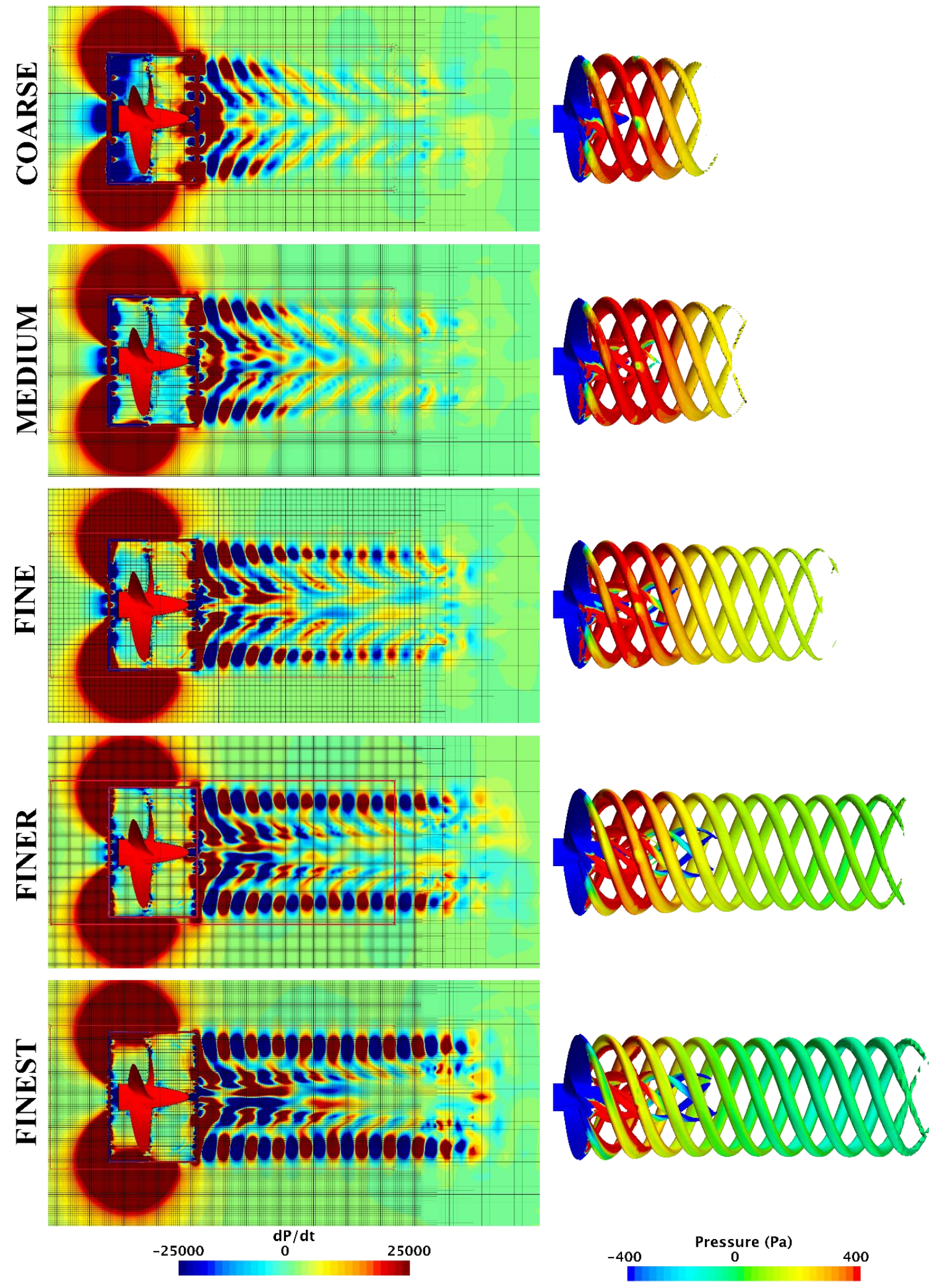

Figure 8. Change in vortex distributions with different grid resolutions (Right figure is coloured by pressure, $Q_{c}=500$ ) 


\subsubsection{Hydroacoustic results}

Figure 9 and Table 6 show the receiver locations and coordinates in the near field, respectively. The origin of the coordinate system was set to the centre of the propeller blades. Receiver 1 (R1) was located in the upstream, whereas R3, R4 and R5 were located in the downstream of the propeller. R2 and R6 were also located on the propeller plane. Since there is no experimental noise data for the selected propeller, the numerical results at R2, R4 and R6 were compared with other numerical studies in the literature, which were conducted by Ianniello et al. (2013a) and Testa et al. (2018). It should mentioned that the numerical results in the reference studies were obtained by using data digitiser software.

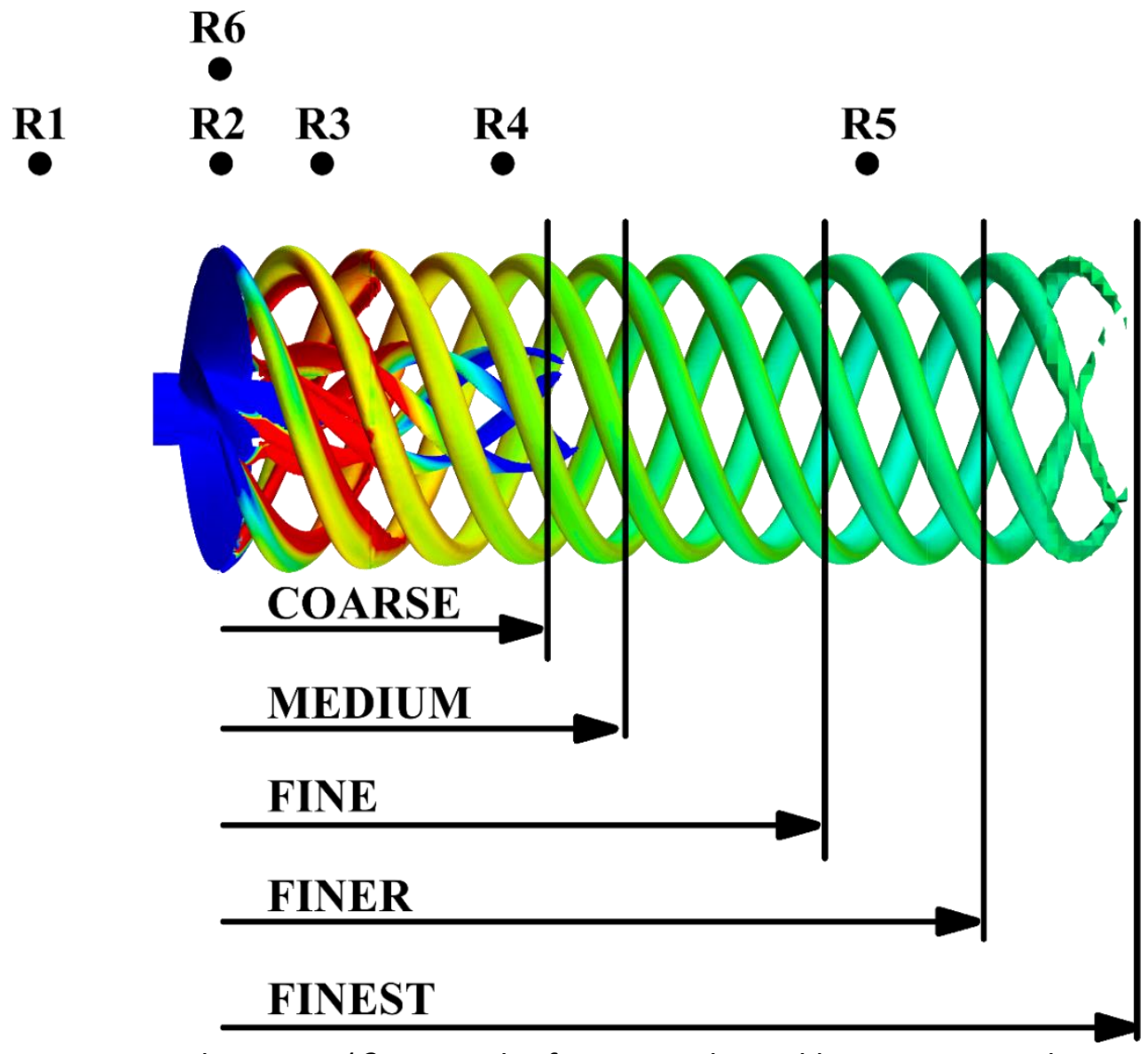

Figure 9. Receiver locations $\left(Q_{c}=500\right.$, the figure is coloured by pressure and not scaled).

Table 5. Receiver coordinates.

\begin{tabular}{|c|c|c|c|}
\hline Receiver & $\mathrm{X}(\mathrm{m})$ & $\mathrm{Y}(\mathrm{m})$ & $\mathrm{Z}(\mathrm{m})$ \\
\hline 1 & 0.1403 & & 0.1703 \\
\hline 2 & 0 & & 0.1703 \\
\hline 3 & -0.0800 & \multirow{2}{*}{0} & 0.1703 \\
\hline 4 & -0.2270 & & 0.1703 \\
\hline 5 & -0.4569 & & 0.1703 \\
\hline 6 & 0 & & 0.2648 \\
\hline
\end{tabular}


As the receivers were positioned in the vicinity of the propeller where the incompressibility assumption is still not visible, the hydrodynamic and hydroacoustic pressures can be compared with each other to show the consistency of the numerical solution. However, with an increase in distance between the noise source and receiver, hydrodynamic pressures tend to deteriorate due to the numerical diffusion. For this reason, the acoustic analogy is required for the prediction of far-field noise since the linear terms of FWH is only dependent on blade shape and hydrodynamic load (Ianniello et al., 2013a; Sezen and Kinaci, 2019).

Figure 10A-C shows the comparison of hydrodynamic and hydroacoustic pressures at R1 with different grid resolutions. At this receiver location, the contribution of linear noise terms (i.e. thickness and loading) to the overall acoustic pressure is dominant, whereas the contribution of nonlinear noise term is almost negligible. The hydrodynamic pressures, which were obtained with different grid resolutions, are compared with each other in Figure 10A. The results show that the amplitude of the hydrodynamic pressure slightly changes with different grid resolutions in Figure 10A. The overall hydroacoustic pressure is purely characterised by BPF (Blade Passage Frequency) at R1 in Figure 10B due to the dominant contribution of linear noise terms. As seen in Figure 10B, the inadequate grid resolution triggers the numerical noise, which is probably induced by sliding interfaces (see Figure 6), and causes deterioration of the overall shape of the acoustic signal. Additionally, a good agreement is found between hydrodynamic and hydroacoustic pressures in Figure 10C. Therefore, the hydrodynamic pressure may be used instead of hydroacoustic pressure at this receiver location to evaluate the propeller URN.
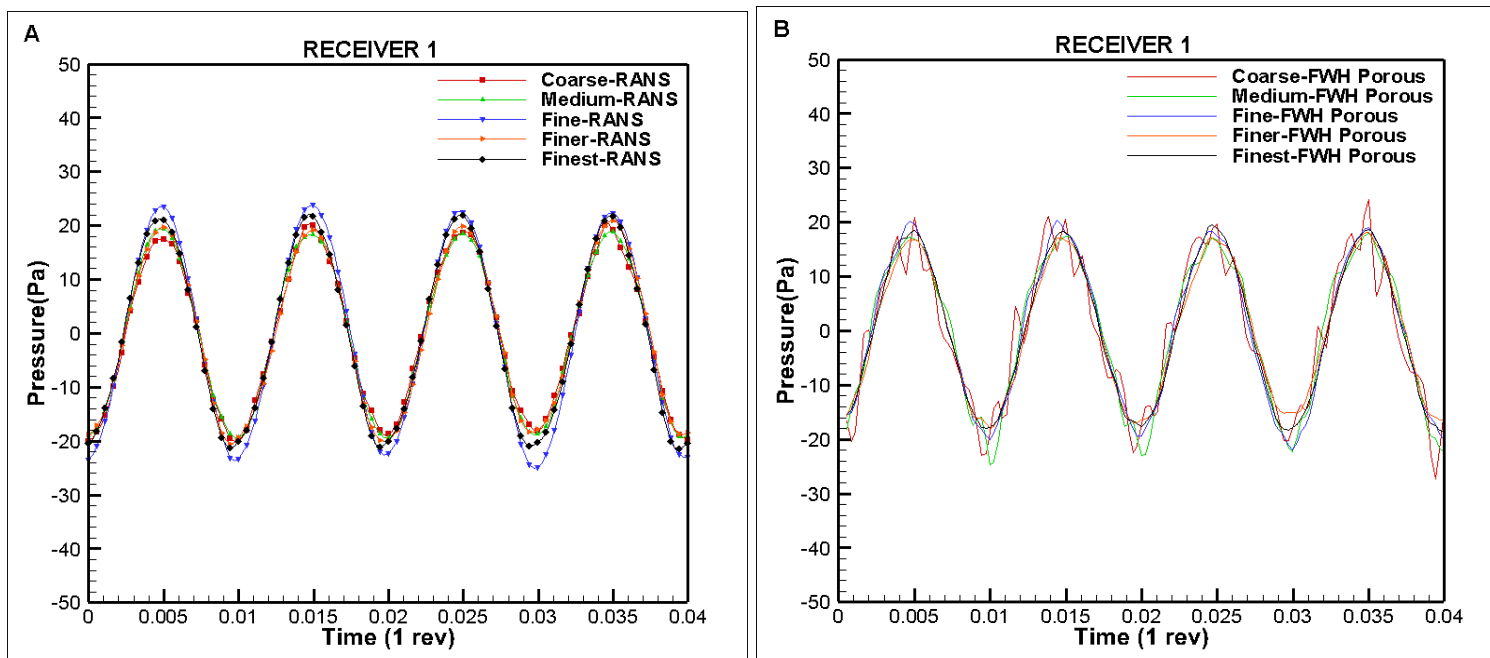


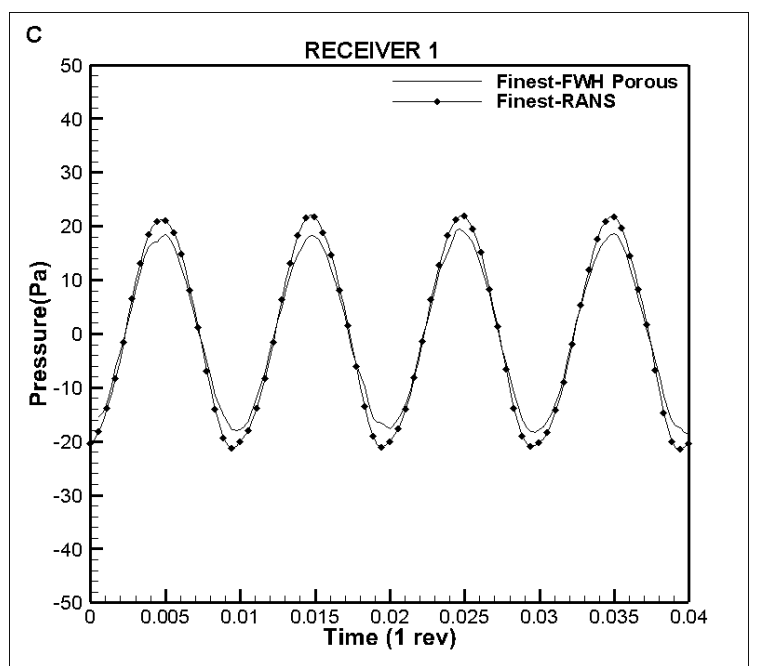

Figure 10. RANS pressures (A), $\mathrm{FW}-\mathrm{H}$ pressures (B) and RANS versus $\mathrm{FW}-\mathrm{H}$ pressures (C) at Receiver 1.

Figure 11 shows the comparison between hydrodynamic and hydroacoustic pressures at R2. Akin to the previous receiver location, the amplitude of the hydrodynamic pressure does not change considerably with the different grid resolutions in Figure $11 \mathrm{~A}$. Unlike the previous receiver location, the overall shape of the acoustic signal is not strongly affected by the numerical noise in Figure 11B. Potentially, the reason could be that R2 is closer to the propeller when compared to R1. Similar to R1, the overall shape of the signal is dominated by the blade harmonics. Furthermore, the amplitude of the acoustic pressure at R2 is found to be higher than R1 due to the decreased distance from the noise source. Figure $11 \mathrm{C}$ shows the comparison of hydrodynamic and hydroacoustic pressures and the agreement is found quite good. Additionally, the numerical results were compared with another numerical study in the literature, which was performed by DES together with porous FW-H equation, by Testa et al. (2018) in Figure 11D. As can be seen in Figure 11D, the present study (i.e. RANS) slightly overestimated the acoustic pressure in comparison to DES solver at the R2. 

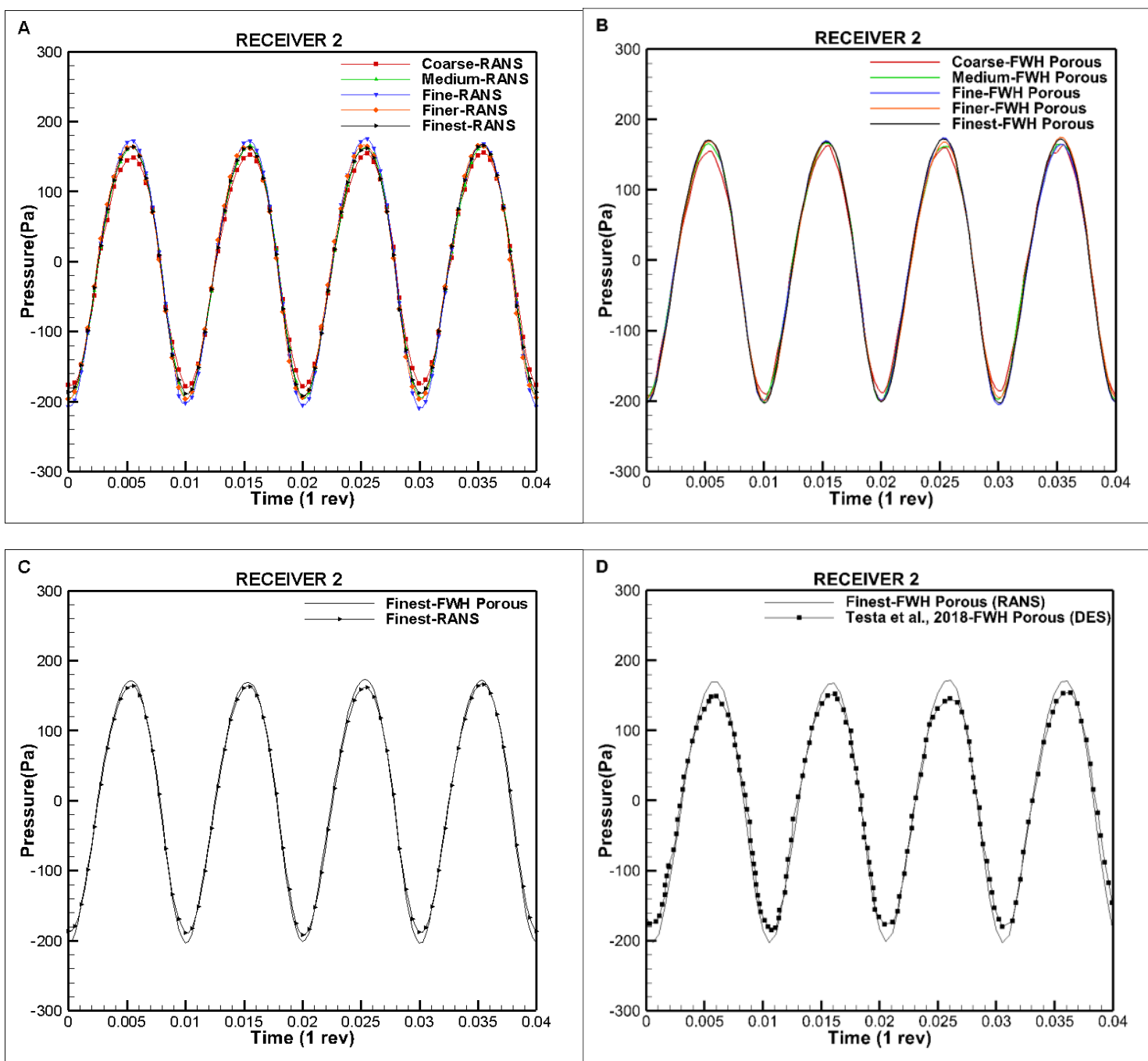

Figure 11. RANS pressures (A), FW-H pressures (B) and RANS versus $\mathrm{FW}-\mathrm{H}$ pressures $(\mathrm{C})$ and comparison of the numerical results (D) at Receiver 2.

The comparison of the pressures is presented in Figure 12 for the R3, which was located in the downstream of the propeller. Similar to R1 and R2, the effects of grid resolution on hydrodynamic pressure is found to be low at R3 (Figure 12A). However, the numerical noise, which is probably created by the sliding interfaces, starts to appear in the hydroacoustic pressures with a decrease in grid resolution in Figure 12B. Thus, the magnitude and shape of the overall acoustic pressure deteriorates, which results in unreliable prediction of propeller URN. As the R3 is still close to the propeller, hydrodynamic and hydroacoustic pressures can be directly compared with each other. As can be seen in Figure 12C, the satisfactory agreement between RANS and porous FW-H pressures are found. 

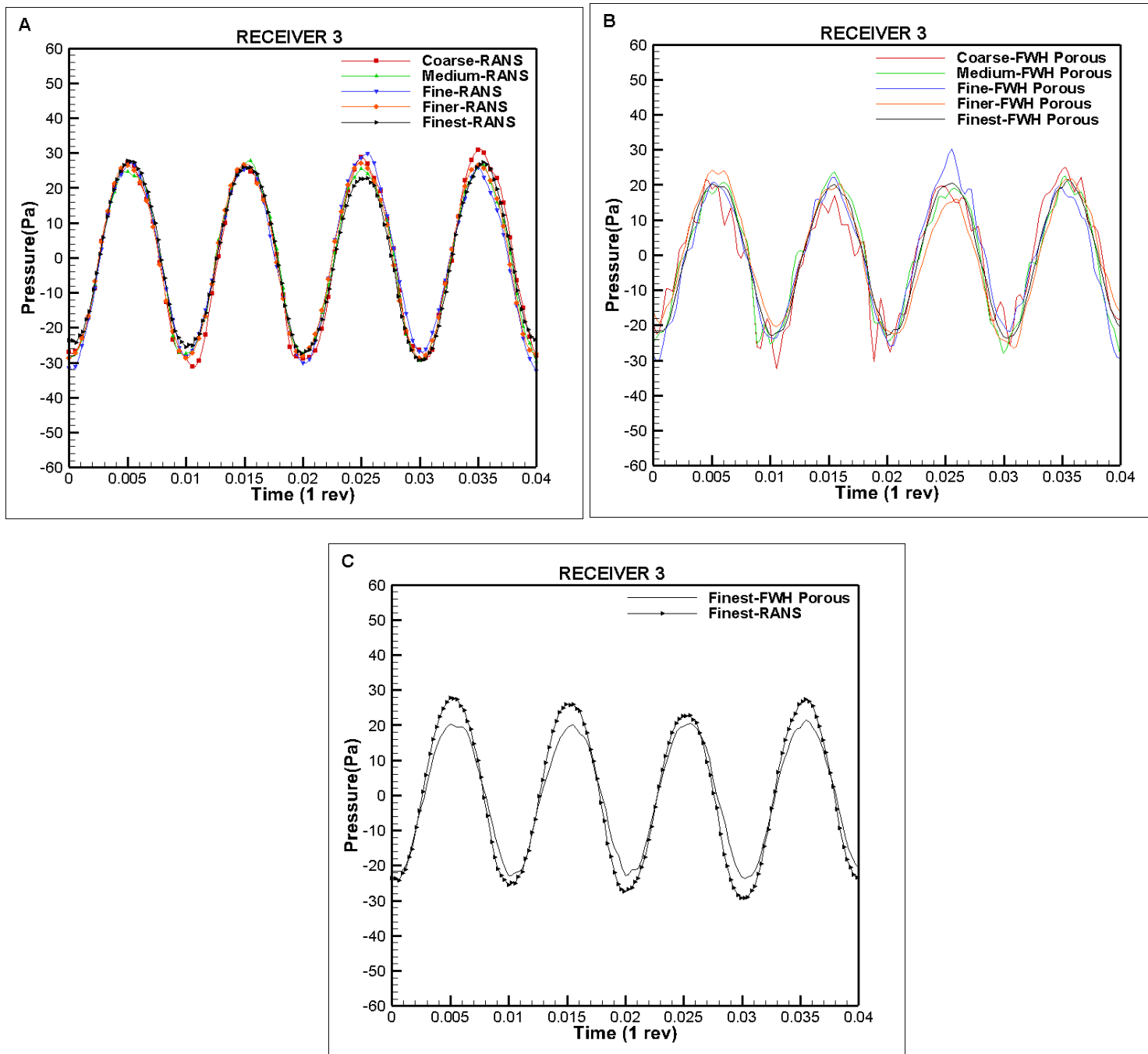

Figure 12. RANS pressures (A), FW-H pressures (B) and RANS versus $\mathrm{FW}-\mathrm{H}$ pressures (C) at Receiver 3.

Increasing the distance from the propeller, hydrodynamic and hydroacoustic pressures start to become completely different at R4 and R5 in terms of magnitude and overall shape of the signal in Figure 13 and Figure 14. The total pressures show a rapid decrease (i.e. from approximately $\mp 30$ to $\mp 1$ ) at R4 in comparison to R3. The variation of hydrodynamic pressures with the different grid resolutions is given in Figure 13A. As shown in Figure 13A, an increase in grid resolution (i.e. more extension of tip vortex in the downstream) does not provide a significant change in the amplitude of hydrodynamic pressures. Hence, these results lead to RANS solver to be questioned even if the numerical diffusion was significantly reduced. The change of hydroacoustic pressures with different grid resolutions is also given in Figure 13B. Similar to hydrodynamic pressures, the magnitude of the acoustic pressures are too small. In other words, a few diameters away from the propeller in the downstream, the total noise tends to disappear, which is not realistic. In the study of Testa et al. (2018), the propeller 
URN was predicted by using DES together with porous FW-H equation at R4. Although the low level of pressure fluctuations coming from the direct DES solution was considered to be a result of the reflection of numerical disturbances from the computational domain in their study, the hydrodynamic and hydroacoustic pressure fluctuations were detected between around $\mp 8$ using the DES method. As depicted in Figure $13 \mathrm{C}$, the RANS method underestimated the hydroacoustic pressures in comparison to DES solver during a propeller revolution. Due to the strong contribution of the nonlinear noise term to the overall acoustic pressure, the overall shape of the signal is no longer modified purely by BPF.
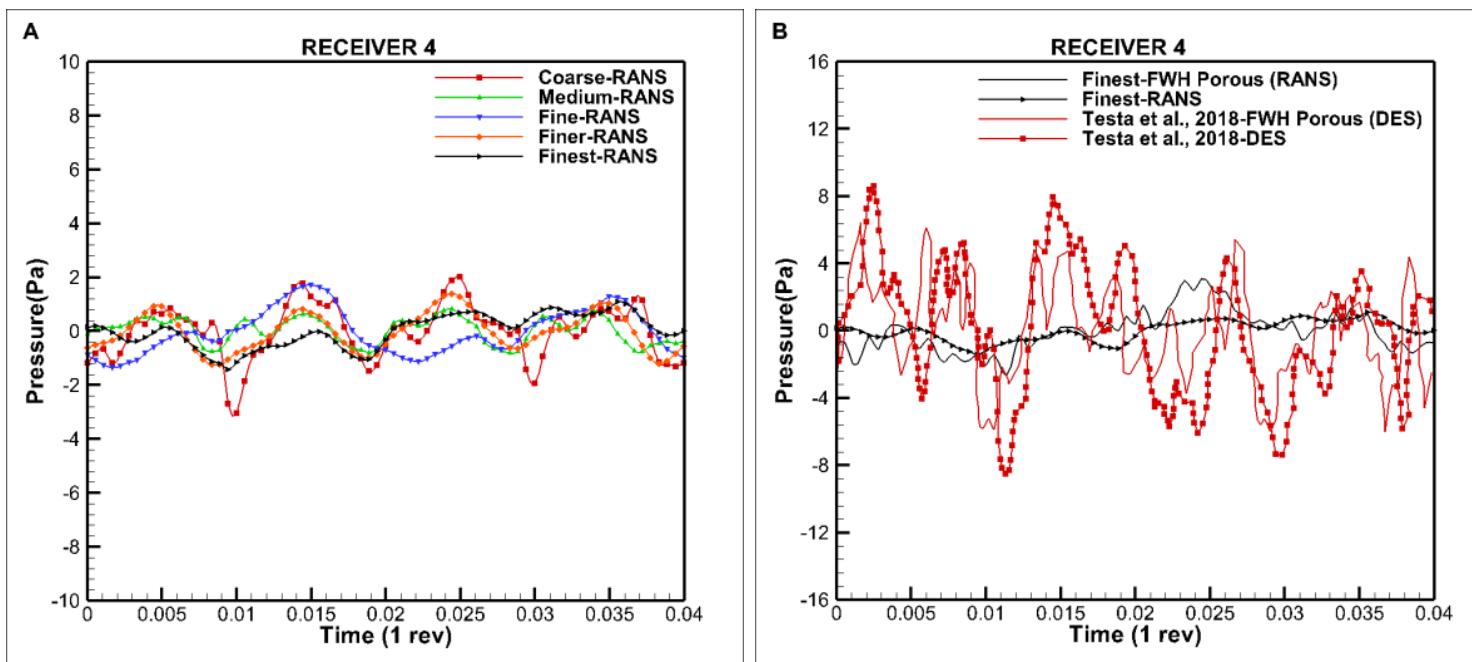

Figure 13. RANS pressures (A), RANS versus FW-H pressures and comparison of the results (B) at Receiver 4

Another comparison of the pressures with different grid resolutions is performed at R5 in Figure 14. Similar to R4, a decrease in numerical diffusion does not give a favourable impact on hydrodynamic and hydroacoustic pressures (Figure 14A and Figure 14B). Additionally, the amplitudes of the pressure signal at R5 are expected to be lower than R4. There could be numerous reasons why an incorrect prediction occurs at R5. This could be caused by the numerical noise induced by the sliding interfaces, mesh transitions, numerical errors, or strong vortex-vortex interactions in the propeller's slipstream. The same issue was also observed in the studies of Lloyd et al. (2015b) and Testa et al. (2018). 

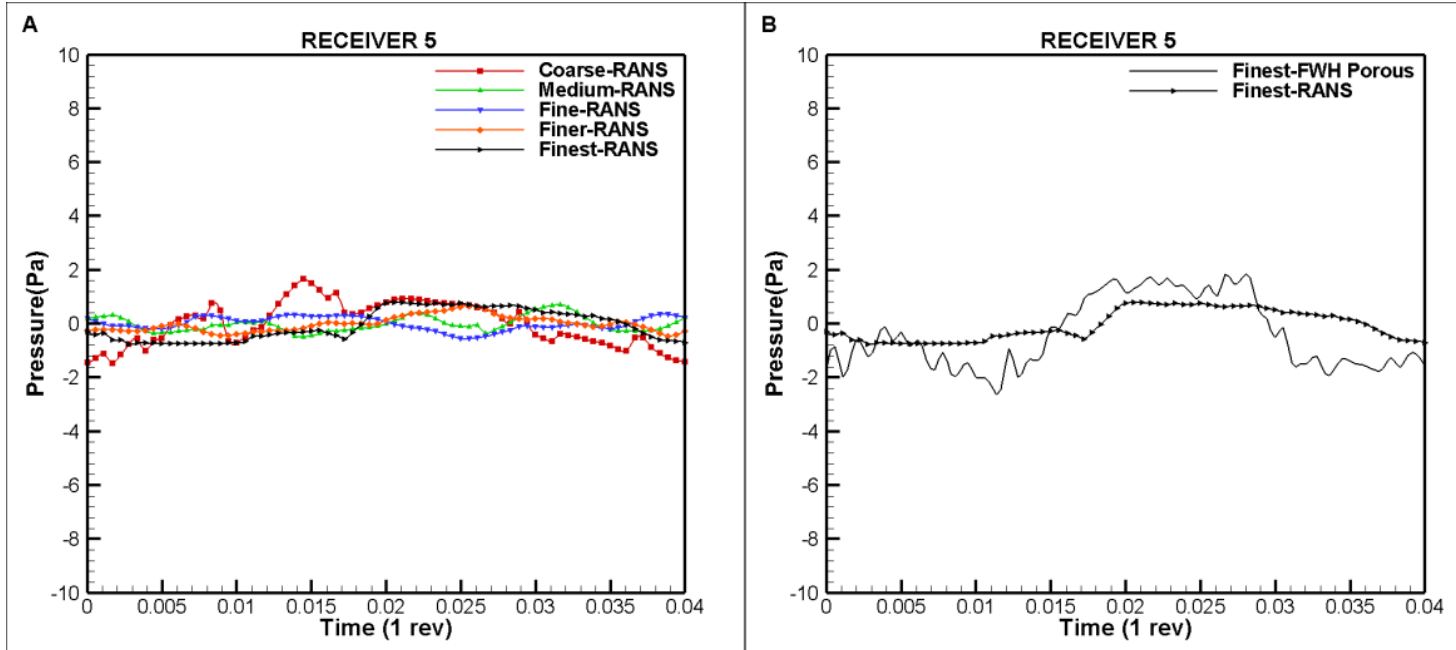

Figure 14. RANS pressures (A), RANS versus FW-H pressures (B) at Receiver 5.

In addition to the comprehensive investigation of the hydrodynamic and hydroacoustic pressures at different receiver locations, the convergence study was also conducted to check the consistency of the solution with different integral surfaces, which are given in Table 2, by using fine grid resolution. Figure $15 \mathrm{~A}, 15 \mathrm{~B}$ and $15 \mathrm{C}$ show the change of hydroacoustic pressures with different porous surfaces at R1, R2 and R3 receiver locations, respectively. As shown in the Figure 15A-C, all of them superimpose each other.
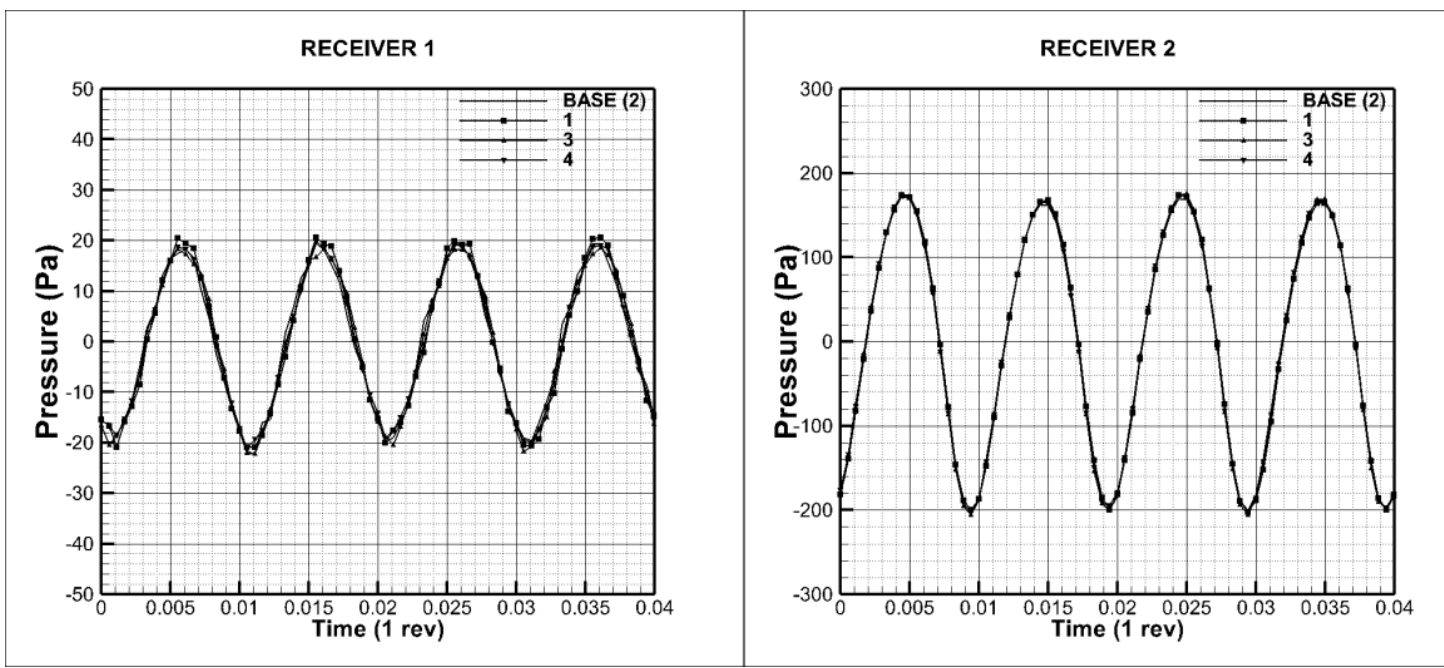


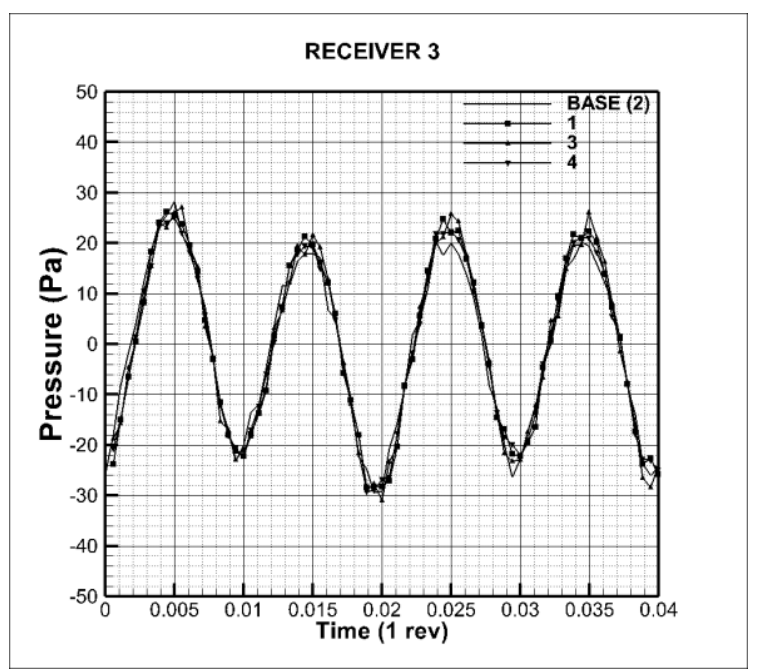

Figure 15. Comparison of hydroacoustic pressures with different integral surfaces at Receiver 1, 2, and 3.

\section{Conclusion}

In this study, the influence of grid resolution on hydroacoustic performance prediction of a marine propeller was investigated under non-cavitating conditions at $\mathrm{J}=0.88$. The numerical results advised some crucial outcomes as follows.

- The hydroacoustic analysis was found more dependent on grid resolution than the prediction of propeller performance characteristics due to the numerical noise induced by the sliding interfaces. Therefore, the grid resolution, which is specifically designed for hydrodynamic purposes, may not be suitable for accurate prediction of the propeller URN.

- Even though the sliding mesh (i.e. RBM) has been widely used for hydrodynamic studies in the literature, this approach suffers from the numerical noise induced by the interfaces. Additionally, this numerical noise issue in the RBM originates from the grid resolution and it is independent of the selected numerical methodology (i.e. RANS, DES and LES). Therefore, overset type grids (also known as Chimera or the overlapping grids) might be more useful method to avoid the additional non-physical noise for hydroacoustic simulations. The reason being, there is a zone where cells overlap between overset and background regions, unlike the sliding interface approach. Nevertheless, it needs to be investigated in a study solely focussed on this subject. 
- The time derivative of the pressure technique can be a useful way to show the numerical noise in the numerical solvers. Additionally, an increase in grid resolution enabled a reduction in the numerical noise, but the results showed that it is not possible to remove it completely.

- The time derivative of the pressure approach can be a more efficient way to determine the accurate extension of the tip vortex in the propeller's slipstream. This approach can be additionally beneficial for further in-depth studies by using advanced models (i.e. DES and LES). From the hydroacoustic point of view, this approach may provide a basis to determine the integral surface location that includes all relevant nonlinear noise contributions generated by vortices as much as possible, particularly for DES and LES methods.

- A decrease in numerical diffusion in the RANS solver provided more extension of the tip vortex in the propeller's slipstream. However, aside from the poor modelling of turbulence, the RANS approach is still inadequate for the more realistic prediction of propeller URN, particularly for the receivers located in the downstream of the propeller. Eventually, the reliable hydroacoustic prediction requires an accurate solution of the flow field by using DES or LES methods.

\section{Acknowledgements}

The first author is sponsored by the Stone Marine Propulsion Ltd of the UK and the University of Strathclyde during his PhD study. Results were obtained using the ARCHIE-WeSt HighPerformance Computer (www.archie-west.ac.uk) based at the University of Strathclyde. The authors are also grateful to CNR-INSEAN, especially Francesco Salvatore, for providing the propeller geometry and experimental results. 


\section{References}

Bensow, R.E., Liefvendahl, M., 2016. An acoustic analogy and scale-resolving flow simulation methodology for the prediction of propeller radiated noise. 31st Symposium on Naval Hydrodynamics, Monterey, California. ss. 1-19.

Celik, I.B., Ghia, U., Roache, P.J., Freitas, C.J., Coleman, H., Raad, P.E., 2008. Procedure for estimation and reporting of uncertainty due to discretization in CFD applications. J. Fluids Eng. Trans. ASME 130, 0780011-0780014. https://doi.org/10.1115/1.2960953

Cianferra, M., Petronio, A., Armenio, V., 2019. Non-linear noise from a ship propeller in open sea condition. Ocean Eng. 191, 106474.

Curle, N., 1955. The influence of solid boundaries upon aerodynamic sound. Proc. R. Soc. London. Ser. A. Math. Phys. Sci. 231, 505-514. https://doi.org/10.1098/rspa.1955.0191

Di Francescantonio, P., 1997. A New Boundary Integral Formulation for the Prediction of Sound Radiation. J. Sound Vib. 202, 491-509.

Duraisamy, K., Baeder, J.D., 2006. Numerical simulation of the effects of spanwise blowing on tip vortex formation. J. Aircr. 43, 996-1006. https://doi.org/10.2514/1.19746

Epps, B.P., 2017. Review of vortex identification methods. AIAA SciTech Forum - 55th AIAA Aerospace Sciences Meeting, Grapevine, Texas. https://doi.org/10.2514/6.2017-0989

Farassat, F., 2007. Derivation of Formulations 1 and 1A of Farassat. Nasa/TM-2007-214853 214853, 1-25.

Feder, D.-F., Abdel-Maksoud, M., 2016. Tracking a Tip Vortex with Adaptive Vorticity Confinement and Hybrid RANS-LES. Open J. Fluid Dyn. 06, 406-429. https://doi.org/10.4236/ojfd.2016.64030

Felli, M., Di Felice, F., Guj, G., Camussi, R., 2006. Analysis of the propeller wake evolution by pressure and velocity phase measurements. Exp. Fluids 41, 441-451. https://doi.org/10.1007/s00348-006-0171-4

Ffowcs Williams, J.E., Hawkings, D.L., 1969. Sound Generation by Turbulence and Surfaces in Arbitrary Motion. Philos. Trans. R. Soc. London. Ser. A, Math. Phys. Sci. 264, 321-342. https://doi.org/10.1098/rsta.1969.0031

Hildebrand, J.A., 2009. Anthropogenic and natural sources of ambient noise in the ocean. Mar. Ecol. Prog. Ser. 395, 5-20. https://doi.org/10.3354/meps08353

Hunt, J.C.R., Wray, A.A., Moin, P., 1988. Eddies, streams, and convergence zones in turbulent flows, Center for Turbulence Research Report CTR-S88, p. 193. https://doi.org/CTR-S88

lanniello, S., De Bernardis, E., 2015. Farassat's formulations in marine propeller hydroacoustics. Int. J. Aeroacoustics 14, 87-103. https://doi.org/10.1260/1475472X.14.1-2.87

lanniello, S., Muscari, R., Di Mascio, A., 2013a. Ship underwater noise assessment by the acoustic analogy. Part I: Nonlinear analysis of a marine propeller in a uniform flow. J. Mar. Sci. Technol. 18, 547-570. https://doi.org/10.1007/s00773-013-0227-0

lanniello, S., Muscari, R., Di Mascio, A., 2013b. Ship underwater noise assessment by the Acoustic Analogy part II: Hydroacoustic analysis of a ship scaled model. J. Mar. Sci. Technol. 19, 52-74. https://doi.org/10.1007/s00773-013-0236-z

lanniello, S., Muscari, R., Di Mascio, A., 2013c. Ship underwater noise assessment by the acoustic analogy, part III: Measurements versus numerical predictions on a full-scale ship. J. Mar. Sci. Technol. 19, 125-142. https://doi.org/10.1007/s00773-013-0228-z

Ianniello, S., Muscari, R., Di Mascio, A., 2012. Hydroacoustic characterization of a marine propeller through the acoustic analogy. Sustain. Marit. Transp. Exploit. Sea Resour. 9911000. https://doi.org/10.1201/b11810-146 
IMO, M.R., 2014. Guidelines for the reduction of underwater noise from commercial shipping to address adverse impacts on marine life, MEPC.1/ Circ.833

ITTC, 2014a. Specialist Committee on Hydrodynamic Noise, Final Report and Recommendations to the 27th ITTC.

ITTC, 2014b. Specialist Committee on Hydrodynamic Noise, Written Discussion at the 27th ITTC.

ITTC, 2008. Recommended Procedures and Guidelines (7.5-03-01-01). Uncertainty Analysis in CFD Verification and Validation Methodology and Procedures.

ITTC, 1999. Recommended Procedures and Guidelines (7.5-03-01-04), CFD-General CFD Verification.

IWC, 2009. Report of the scientific committee. Journal of Cetacean Research and Management, 11 , Section 12. Environmental Concerns, 46-47.

Jang, C.M., Furukawa, M., Inoue, M., 2001. Analysis of vortical flow field in a propeller fan by LDV measurements and LES-Part II: Unsteady nature of vortical flow structures due to tip vortex breakdown. J. Fluids Eng. Trans. ASME 123, 755-761. https://doi.org/10.1115/1.1412566

Jeong, J., Hussain, F., 1995. On the identification of a vortex. J. Fluid Mech. 285, 69-94. https://doi.org/10.1017/S0022112095000462

Jones, D.A., Clarke, D.B., 2010. Fluent code simulation of flow around a naval hull: the DTMB 5415. Maritime Platforms Division Defence Science and Technology Organisation, Report Number: DSTO-TR-2465

Kehr, Y.Z., Kao, J.H., 2011. Underwater acoustic field and pressure fluctuation on ship hull due to unsteady propeller sheet cavitation. J. Mar. Sci. Technol. 16, 241-253. https://doi.org/10.1007/s00773-011-0131-4

Kellett, P., Turan, O., Incecik, A., 2013. A study of numerical ship underwater noise prediction. Ocean Eng. 66, 113-120. https://doi.org/10.1016/j.oceaneng.2013.04.006

Kolář, V., 2007. Vortex identification: New requirements and limitations. Int. J. Heat Fluid Flow 28, 638-652. https://doi.org/10.1016/j.ijheatfluidflow.2007.03.004

Li, D.Q., Hallander, J., Johansson, T., 2018. Predicting underwater radiated noise of a full scale ship with model testing and numerical methods. Ocean Eng. 161, 121-135. https://doi.org/10.1016/j.oceaneng.2018.03.027

Lidtke, Artur K., 2017. Predicting radiated noise of marine propellers using acoustic analogies and hybrid Eulerian-Lagrangian cavitation models, PhD Thesis. University of Southampton.

Lidtke, A.K., Lloyd, T., Vaz, G., 2019. Acoustic modelling of a propeller subject to non-uniform inflow. Proc. 6th Int. Symp. Mar. Propulsors, SMP'19, Rome, Italy.

Lidtke, A.K., Turnock, S.R., Humphrey, V.F., 2015. Use of Acoustic Analogy for Marine Propeller Noise Characterisation, 4th International Symposium on Marine Propulsors SMP'15, Austin, Texas, USA.

Lighthill, M.J., A, P.R.S.L., 1952. On sound generated aerodynamically I. General theory. Proc. R. Soc. London. Ser. A. Math. Phys. Sci. 211, 564-587. https://doi.org/10.1098/rspa.1952.0060

Lloyd, T.P., Lidtke, A.K., Rijpkema, D., Van Wijngaarden, E., Turnock, S.R., Humphrey, V.F., 2015a. Using the FW-H equation for hydroacoustics of propellers. 15th Numer. Towing Tank Symp. (NuTTS) Cortona,Italy.

Lloyd, T.P., Rijpkema, D.R., van Wijngaarden, E., 2015b. Marine propeller acoustic modelling: comparing CFD results with an acoustic analogy method. Fourth Int. Symp. Mar. 
Propulsors SMP'15, Austin, Texas.

Lloyd, T.P., Rijpkema, D.R., van Wijngaarden, E., 2014. Implementing the Ffowcs WilliamsHawkings acoustic analogy into a viscous CFD solver. 17th Numer. Towing Tank Symp. (NuTTS),Marstrand, Sweden.

Lyrintzis, A.S., 2003. Surface Integral Methods in Computational Aeroacoustics-From the (CFD) Near-Field to the (Acoustic) Far-Field. Int. J. Aeroacoustics 2, 95-128. https://doi.org/10.1260/147547203322775498

Merchant, N.D., Blondel, P., Dakin, D.T., Dorocicz, J., 2012. Averaging underwater noise levels for environmental assessment of shipping. J. Acoust. Soc. Am. 132, EL343-EL349. https://doi.org/10.1121/1.4754429

Moussa, K., 2014. Computational Modeling of Propeller Noise NASA SR-7A. Master Thesis. Waterloo,Ontario, Canada.

Muscari, R., Di Mascio, A., Verzicco, R., 2013. Modeling of vortex dynamics in the wake of a marine propeller. Comput. Fluids 73, 65-79. https://doi.org/10.1016/j.compfluid.2012.12.003

Najafi-Yazdi, A., Brés, G.A., Mongeau, L., 2010. An acoustic analogy formulation for moving sources in uniformly moving media. Proc. R. Soc. A Math. Phys. Eng. Sci. 467, 144-165. https://doi.org/10.1098/rspa.2010.0172

Nitzkorski, Z., 2015. A novel porous Ffowcs-Williams and Hawkings acoustic methodology for complex geometries, PhD Thesis. Faculty of the Graduate School of the University of Minnesota.

Ramponi, R., Blocken, B., 2012. CFD simulation of cross-ventilation flow for different isolated building configurations: Validation with wind tunnel measurements and analysis of physical and numerical diffusion effects. J. Wind Eng. Ind. Aerodyn. 104-106, 408-418. https://doi.org/10.1016/j.jweia.2012.02.005

Richardson, L.F., 1911. IX. The approximate arithmetical solution by finite differences of physical problems involving differential equations, with an application to the stresses in a masonry dam. Philos. Trans. R. Soc. London. Ser. A, Contain. Pap. a Math. or Phys. Character 210, 307-357. https://doi.org/10.1098/rsta.1911.0009

Roache, P.J., 1998. Verification of codes and calculations. AIAA J. 36, 696-702. https://doi.org/10.2514/2.457

Salvatore, F., Testa, C., Ianniello, S., Pereira, F., 2006. Theoretical Modelling of Unsteady Cavitation and Induced Noise, Sixth International Symposium on Cavitation CAV2006, Wageningen, The Netherlands. ss. 1-13.

Seol, H., 2013. Time domain method for the prediction of pressure fluctuation induced by propeller sheet cavitation: Numerical simulations and experimental validation. Ocean Eng. 72, 287-296.

Seol, H., Jung, B., Suh, J.C., Lee, S., 2002. Prediction of non-cavitating underwater propeller noise. J. Sound Vib. 257, 131-156. https://doi.org/10.1006/jsvi.2002.5035

Seol, H., Suh, J.C., Lee, S., 2005. Development of hybrid method for the prediction of underwater propeller noise. J. Sound Vib. 288, 345-360. https://doi.org/10.1016/j.jsv.2005.01.015

Sezen, S., Kinaci, O.K., 2019. Incompressible flow assumption in hydroacoustic predictions of marine propellers. Ocean 106138. https://doi.org/10.1016/j.oceaneng.2019.106138

Sezen, S., Atlar, M., Fitzsimmons, P., Sasaki, N., Tani. G., Yilmaz, N., Aktas, B., 2020. NumericalCavitation Noise Prediction of a Benchmark Research Vessel Propeller. Ocean 
Engineering,Volume 211, 107549.

Star CCM+, 2019. User Guide.

Testa, C., Greco, L., 2018. Prediction of submarine scattered noise by the acoustic analogy. J. Sound Vib. 426, 186-218. https://doi.org/10.1016/j.jsv.2018.04.011

Testa, C., lanniello, S., Salvatore, F., Gennaretti, M., 2008. Numerical approaches for hydroacoustic analysis of marine propellers. J. Sh. Res. 52, 57-70.

Testa, C., Porcacchia, F., Greco, L., Muscari, R., 2018. Effectiveness of Boundary Element Method Hydrodynamic Data for Propeller Hydroacoustics, A. Yucel Odabasi Colloquium Series 3rd International Meeting-Progress in Propeller Cavitation and Its Consequences: Experimental and Computational Methods for Predictions, Istanbul, Turkey. ss. 77-85.

Urick, R.J., 1983. Principles of Underwater Sound, 3rd Edition. McGraw-Hill. New York.

Wei, Y., Shen, Y., Jin, S., Hu, P., Lan, R., Zhuang, S., Liu, D., 2016. Scattering effect of submarine hull on propeller non-cavitation noise. J. Sound Vib. 370, 319-335. https://doi.org/10.1016/j.jsv.2016.01.027

Zhu, Z., 2014. Characteristic correlation between propellers cavitating wake and cavitation noise. Appl. Acoust. 81, 31-39. https://doi.org/10.1016/j.apacoust.2014.02.004 